\title{
Production of doubly charged scalars from the decay of a heavy SM-like Higgs boson in the Higgs Triplet Model
}

\author{
A.G. Akeroyd* and S. Morettit \\ ${ }^{1}$ School of Physics and Astronomy, University of Southampton, \\ Highfield, Southampton SO17 1BJ, United Kingdom, and \\ ${ }^{2}$ Particle Physics Department, Rutherford Appleton Laboratory, \\ Chilton, Didcot, Oxon OX11 0QX, United Kingdom
}

\begin{abstract}
The Higgs Triplet Model (HTM) of neutrino mass generation predicts the existence of doubly charged Higgs bosons $\left(H^{ \pm \pm}\right)$. In the HTM a scalar eigenstate $\left(H_{2}\right)$ is dominantly composed of the scalar field from the isospin doublet, and could be significantly heavier than $H^{ \pm \pm}$. Such a scenario would allow the possibility of a large branching ratio for the decay $\mathrm{H}_{2} \rightarrow \mathrm{H}^{++} \mathrm{H}^{--}$. From the production mechanism of gluon-gluon fusion, $g g \rightarrow H_{2}$, the above decay mode would give rise to pair production of doubly charged Higgs bosons $\left(H^{++} H^{--}\right)$with a cross section which could be significantly larger than the cross sections for the standard production mechanisms $q \bar{q} \rightarrow \gamma, Z \rightarrow H^{++} H^{--}$and $q^{\prime} \bar{q} \rightarrow W \rightarrow H^{ \pm \pm} H^{\mp}$. We discuss the phenomenological consequences for the ongoing searches for $H^{ \pm \pm}$at the Tevatron and at the LHC.
\end{abstract}

PACS numbers: 14.80.Fd, 12.60.Fr

*Electronic address: a.g.akeroyd@soton.ac.uk

${ }^{\dagger}$ Electronic address: S.Moretti@soton.ac.uk 


\section{INTRODUCTION}

The established evidence that neutrinos oscillate and possess a small mass below the electron volt (eV) scale [1] necessitates physics beyond the Standard Model (SM), which could manifest itself at the CERN Large Hadron Collider (LHC) and the Fermilab Tevatron, and/or in low energy experiments which search for lepton flavour violation (LFV) [2]. Consequently, models of neutrino mass generation which can be probed at present and forthcoming experiments are of great phenomenological interest.

Neutrinos may obtain mass via the vacuum expectation value (vev) of a neutral Higgs boson in an isospin triplet representation [3 7]. A particularly simple implementation of this mechanism of neutrino mass generation is the "Higgs Triplet Model" (HTM) in which the SM Lagrangian is augmented solely by a $S U(2)$ triplet of scalar particles $\Delta$ with hypercharge $Y=2[3,6]$, 7] . In the HTM, neutrinos acquire a Majorana mass given by the product of a triplet Yukawa coupling $\left(h_{i j}\right.$, with $\left.i, j=e, \mu, \tau\right)$ and a triplet vev $\left(v_{\Delta}\right)$. Consequently, there is a direct connection between $h_{i j}$ and the neutrino mass matrix, which gives rise to phenomenological predictions for processes which depend on $h_{i j}$. A distinctive signal of the HTM would be the observation of doubly charged Higgs bosons $\left(H^{ \pm \pm}\right)$whose mass $\left(M_{H^{ \pm \pm}}\right)$may be of the order of the electroweak scale. Such particles could be produced with sizeable rates at hadron colliders via the processes $q \bar{q} \rightarrow H^{++} H^{--}[8]$ [2] and $q^{\prime} \bar{q} \rightarrow$ $H^{ \pm \pm} H^{\mp}\left[\right.$ [8, 13, 14] . The first searches for $H^{ \pm \pm}$at a hadron collider were carried out at the Fermilab Tevatron, assuming the production channel $q \bar{q} \rightarrow H^{++} H^{--}$and decay $H^{ \pm \pm} \rightarrow$ $\ell_{i}^{ \pm} \ell_{j}^{ \pm}$. The mass limits $M_{H^{ \pm \pm}}>110 \rightarrow 150 \mathrm{GeV}$ [15 18] were derived, with the strongest limits being for $\ell=e, \mu$ [15 17]. The branching ratios (BRs) for $H^{ \pm \pm} \rightarrow \ell_{i}^{ \pm} \ell_{j}^{ \pm}$depend on $h_{i j}$ and are predicted in the HTM in terms of the parameters of the neutrino mass matrix [14, 19, 20]. Detailed quantitative studies of $\mathrm{BR}\left(H^{ \pm \pm} \rightarrow \ell_{i}^{ \pm} \ell_{j}^{ \pm}\right)$(and $\mathrm{BR}\left(H^{ \pm} \rightarrow \ell_{i}^{ \pm} \nu\right)$ ) in the HTM have been performed in [21 26] ] with particular emphasis given to their sensitivity to the Majorana phases and the absolute neutrino mass i.e. parameters which cannot be probed in neutrino oscillation experiments. Simulations of the detection prospects of $H^{ \pm \pm}$at the LHC with $\sqrt{s}=14 \mathrm{TeV}$ previously focussed on $q \bar{q} \rightarrow \gamma^{*}, Z^{*} \rightarrow H^{++} H^{--}$only [27, 28], but recent studies now include the mechanism $q^{\prime} \bar{q} \rightarrow H^{ \pm \pm} H^{\mp}$ [24, 25, 29]. The first search for $H^{ \pm \pm}$at the LHC with $\sqrt{s}=7 \mathrm{TeV}$ [30] has recently been performed for both production mechanisms $q \bar{q} \rightarrow H^{++} H^{--}$and $q \overline{q^{\prime}} \rightarrow H^{ \pm \pm} H^{\mp}$, for the decay channels $H^{ \pm \pm} \rightarrow \ell_{i}^{ \pm} \ell_{j}^{ \pm}$and $H^{ \pm} \rightarrow \ell_{i}^{ \pm} \nu$ (where $\left.i, j=e, \mu, \tau\right)$.

In the HTM there are two electrically neutral mass eigenstates which are CP-even scalars. These are denoted by $H_{1}$ and $H_{2}$, with $M_{H_{1}}<M_{H_{2}}$. One of the eigenstates is dominantly composed of the isospin doublet field (and plays the role of the SM Higgs boson) while the other eigenstate is dominantly composed of the real part of the neutral triplet field. The mixing angle is small because it depends on the small ratio $v_{\Delta} / v<0.03$ (where $v=246 \mathrm{GeV}$, the vev of the doublet field). In phenomenological studies of the HTM it is usually assumed that the lighter eigenstate $H_{1}$ is the one which is dominantly composed of the isospin doublet field. Therefore the phenomenology of $H_{1}$ is more or less identical to that of the SM-Higgs boson. The converse case of the heavier eigenstate $H_{2}$ being the one which is dominantly composed of the isospin doublet field is possible in the HTM, and has been mentioned in [31 33]. However, no detailed study of the phenomenology of $H_{2}$ in such a scenario has been carried out. Importantly, if $M_{\mathrm{H}_{2}}>2 M_{\phi}$ [33] (where $\phi$ is one of the dominantly triplet eigenstates $H_{1}, A^{0}, H^{ \pm}, H^{ \pm \pm}$) then new decay channels for $H_{2}$ would be possible. This would give rise to a phenomenology of $\mathrm{H}_{2}$ which differs somewhat from that of the SM 
Higgs boson. In this work we focus on the case of $M_{H_{2}}>2 M_{H^{ \pm \pm}}$, because a new production mechanism for $H^{ \pm \pm}$would be possible, namely gluon-gluon fusion $g g \rightarrow H_{2}$ followed by decay $H_{2} \rightarrow H^{++} H^{--}$. The case of $M_{H_{2}}>2 M_{H^{ \pm \pm}}$necessarily requires $M_{H_{2}} \gtrsim 200 \mathrm{GeV}$, a mass region which is now being probed for the first time by the LHC for the decay channels to SM particles, $H_{2} \rightarrow W W$ and $H_{2} \rightarrow Z Z$ [34, 35].

Our work is organised as follows. In section II we describe the theoretical structure of the HTM. In section III the parameter space for $M_{H_{2}}>2 M_{\phi}$ (where $\phi$ is one of $H_{1}, A^{0}, H^{ \pm}, H^{ \pm \pm}$) is described. In section IV the formulae for the decay widths of $H_{2} \rightarrow H^{++} H^{--}, H^{+} H^{-}, A^{0} A^{0}, H_{1} H_{1}$ are presented. Section $\mathrm{V}$ contains a numerical analysis of the magnitude of the branching ratios of the above channels, as well as a quantitative study of the cross section for pair production of $H^{ \pm \pm}$via $g g \rightarrow H_{2}$, with decay $H_{2} \rightarrow H^{++} H^{--}$. Conclusions are given in section VI.

\section{THE HIGGS TRIPLET MODEL}

In the HTM [3, 6, 7] a $I=1, Y=2$ complex $S U(2)_{L}$ isospin triplet of scalar fields is added to the SM Lagrangian. Such a model can provide a Majorana mass for the observed neutrinos without the introduction of a right-handed neutrino via the gauge invariant Yukawa interaction:

$$
\mathcal{L}=h_{i j} \psi_{i L}^{T} C i \tau_{2} \Delta \psi_{j L}+h . c
$$

Here $h_{i j}(i, j=e, \mu, \tau)$ is a complex and symmetric coupling, $C$ is the Dirac charge conjugation operator, $\tau_{2}$ is a Pauli matrix, $\psi_{i L}=\left(\nu_{i}, \ell_{i}\right)_{L}^{T}$ is a left-handed lepton doublet, and $\Delta$ is a $2 \times 2$ representation of the $Y=2$ complex triplet fields:

$$
\Delta=\left(\begin{array}{cc}
\delta^{+} / \sqrt{2} & \delta^{++} \\
\delta^{0} & -\delta^{+} / \sqrt{2}
\end{array}\right)
$$

A non-zero triplet vacuum expectation value $\left\langle\Delta^{0}\right\rangle$ gives rise to the following mass matrix for neutrinos:

$$
m_{i j}=2 h_{i j}\left\langle\Delta^{0}\right\rangle=\sqrt{2} h_{i j} v_{\Delta}
$$

The necessary non-zero $v_{\Delta}$ arises from the minimisation of the most general $S U(2) \otimes U(1)_{Y}$ invariant Higgs potential, which is written as follows [19, 20] (with $\left.\Phi=\left(\phi^{+}, \phi^{0}\right)^{T}\right)$ :

$$
\begin{aligned}
V(H, \Delta)= & -m_{H}^{2} H^{\dagger} H+\lambda\left(H^{\dagger} H\right)^{2}+M_{\Delta}^{2} \operatorname{Tr} \Delta^{\dagger} \Delta+\left(\mu H^{T} i \sigma_{2} \Delta^{\dagger} H+\text { h.c. }\right) \\
& +\lambda_{1}\left(H^{\dagger} H\right) \operatorname{Tr} \Delta^{\dagger} \Delta+\lambda_{2}\left(\operatorname{Tr} \Delta^{\dagger} \Delta\right)^{2}+\lambda_{3} \operatorname{Tr}\left(\Delta^{\dagger} \Delta\right)^{2}+\lambda_{4} H^{\dagger} \Delta^{\dagger} H(4)
\end{aligned}
$$

Here $m_{H}^{2}<0$ in order to ensure $\left\langle\phi^{0}\right\rangle=v / \sqrt{2}$ which spontaneously breaks $S U(2) \otimes U(1)_{Y}$ to $U(1)_{Q}$, and $M_{\Delta}^{2}(>0)$ is the mass term for the triplet scalars. In the model of GelminiRoncadelli [36] the term $\mu\left(\Phi^{T} i \tau_{2} \Delta^{\dagger} \Phi\right)$ is absent, which leads to spontaneous violation of lepton number for $M_{\Delta}^{2}<0$. The resulting Higgs spectrum contains a massless triplet scalar (a Majoron, $J$ ) and another light scalar $\left(H^{0}\right)$. Pair production via $e^{+} e^{-} \rightarrow H^{0} J$ would give a large contribution to the invisible width of the $Z$ boson and this model was excluded at the CERN Large Electron Positron Collider (LEP). The inclusion of the term $\mu\left(\Phi^{T} i \tau_{2} \Delta^{\dagger} \Phi\right)$ explicitly breaks lepton number when $\Delta$ is assigned $L=2$, and eliminates 
the Majoron [3, 6, 7]. Thus the scalar potential in eq. (4) together with the triplet Yukawa interaction of eq. (11) lead to a phenomenologically viable model of neutrino mass generation. For small $v_{\Delta} / v$, the expression for $v_{\Delta}$ resulting from the minimisation of $V$ is:

$$
v_{\Delta} \simeq \frac{\mu v^{2}}{\sqrt{2}\left(M_{\Delta}^{2}+v^{2}\left(\lambda_{1}+\lambda_{4}\right) / 2\right)}
$$

For large $M_{\Delta}$ compared to $v$ one has $v_{\Delta} \simeq \mu v^{2} / \sqrt{2} M_{\Delta}^{2}$, which is sometimes referred to as the "Type II seesaw mechanism" and would naturally lead to a small $v_{\Delta}$. Recently there has been much interest in the scenario of light triplet scalars $(M \approx v)$, (especially the distinctive doubly charged scalar, $H^{ \pm \pm}$), within the discovery reach of the LHC, for which eq. (5) leads to $v_{\Delta} \approx \mu$. In extensions of the HTM the term $\mu\left(\Phi^{T} i \tau_{2} \Delta^{\dagger} \Phi\right)$ may arise in various ways: i) it can be generated at tree level via the vev of a Higgs singlet field [37]; ii) it can arise at higher orders in perturbation theory [20]; iii) it can originate in the context of extra dimensions [19]; iv) it can arise in models with an additional heavy scalar triplet [38].

Some phenomenological studies focus on a simplified scalar potential (e.g. Ref. [24]) in which the quartic couplings $\lambda_{i}$ (where $i=1,2,3,4$ ) involving the triplet field $\Delta$ are neglected. The resulting scalar potential then depends on four parameters $\left(-m_{H}^{2}, \lambda, \mu\right.$, $\left.M_{\Delta}\right)$, but only three parameters are independent because the VEV for the doublet field $(v=246 \mathrm{GeV})$ is fixed by the mass of $W^{ \pm}$. The three independent parameters are usually chosen as $\lambda, v_{\Delta}, M_{\Delta}$ or $\lambda, v_{\Delta}, \mu$ (see eq. (5) ). The inclusion of $\lambda_{i}$ generates additional trilinear and quartic couplings among the scalar mass eigenstates. The terms with $\lambda_{1}$ and $\lambda_{4}$, which involve both triplet and doublet fields, are of particular interest because they can give a sizeable contribution to the masses of the scalar eigenstates (see below). A detailed study of the theoretical constraints on the scalar potential (e.g. vacuum stability, unitarity and perturbativity) has been carried out in 33 .

An upper limit on $v_{\Delta}$ can be obtained from considering its effect on the parameter $\rho\left(=M_{W}^{2} / M_{Z}^{2} \cos ^{2} \theta_{W}\right)$. In the SM $\rho=1$ at tree-level, while in the HTM one has (where $\left.x=v_{\Delta} / v\right)$ :

$$
\rho \equiv 1+\delta \rho=\frac{1+2 x^{2}}{1+4 x^{2}}
$$

The measurement $\rho \approx 1$ leads to the bound $v_{\Delta} / v \lesssim 0.03$, or $v_{\Delta} \lesssim 8 \mathrm{GeV}$. Production mechanisms which depend on $v_{\Delta}$ (i.e. $p p \rightarrow W^{ \pm *} \rightarrow W^{\mp} H^{ \pm \pm}$and fusion via $W^{ \pm *} W^{ \pm *} \rightarrow$ $\left.H^{ \pm \pm}[12,39]\right)$ are not competitive with the processes $q \bar{q} \rightarrow H^{++} H^{--}$and $\bar{q} q^{\prime} \rightarrow H^{ \pm \pm} H^{\mp}$ at the energies of the Fermilab Tevatron, but such mechanisms could be the dominant source of $H^{ \pm \pm}$at the LHC if $v_{\Delta}=\mathcal{O}(1 \mathrm{GeV})$ and $M_{H^{ \pm \pm}}>500 \mathrm{GeV}$. At the 1-loop level, $v_{\Delta}$ must be renormalised and explicit analyses lead to bounds on its magnitude similar to the above bound from the tree-level analysis, e.g. see [40, 41].

The scalar eigenstates in the HTM are as follows: i) the charged scalars $H^{ \pm \pm}$and $H^{ \pm}$; ii) the CP-even neutral scalars $H_{1}$ and $H_{2}$; iii) a CP-odd neutral scalar $A^{0}$. The doubly charged $H^{ \pm \pm}$is entirely composed of the triplet scalar field $\Delta^{ \pm \pm}$, while the remaining eigenstates are in general mixtures of the doublet and triplet fields. However, such mixing is proportional to the triplet vev, and hence small even if $v_{\Delta}$ assumes its largest value of a few $\mathrm{GeV} .{ }^{1}$

\footnotetext{
${ }^{1}$ A large mixing angle is possible in the CP-even sector provided that $M_{H_{1}} \sim M_{H_{2}}$ 31 33 .
} 


\section{SCENARIO OF A HEAVY SM-LIKE HIGGS BOSON $\left(H_{2}\right)$ IN THE HTM}

In the HTM there are two CP-even mass eigenstates, which we denote by $H_{1}$ and $H_{2}$ (where both $M_{H_{2}}>M_{H_{1}}$ and $M_{H_{1}}>M_{H_{2}}$ are possible). Their compositions in terms of the original fields of the Lagrangian are as follows:

$$
H_{2}=\cos \theta_{0} h^{0}+\sin \theta_{0} \Delta^{0} \quad H_{1}=-\sin \theta_{0} h^{0}+\cos \theta_{0} \Delta^{0}
$$

Here $h^{0}$ is the real part of the electrically neutral doublet field $\phi^{0}$, and $\Delta^{0}$ is the real part of the electrically neutral triplet field $\delta^{0}$. The mixing angle $\theta_{0}$ is very small, ${ }^{2}$ being of order 0.03 at most $\left(\sin \theta_{0} \sim v_{\Delta} / v\right)$. Hence $H_{2}$ is essentially composed of the doublet field $h^{0}$, with couplings to the fermions and gauge bosons which are almost identical to those of the SM Higgs boson, while $H_{1}$ is mainly composed of the triplet field $\Delta^{0}$.

The explicit expression for the $2 \times 2 \mathrm{CP}$-even scalar mass matrix for the scalar potential in eq. (41) is given in several works e.g. [31-33]. Neglecting the small off-diagonal elements in this mass matrix, the approximate expressions for the squared masses of $H_{1}$ and $H_{2}$ are as follows:

$$
\begin{array}{r}
M_{H_{2}}^{2}=2 \lambda v^{2} \\
M_{H_{1}}^{2}=M_{\Delta}^{2}+\left(\frac{\lambda_{1}}{2}+\frac{\lambda_{4}}{2}\right) v^{2}+3\left(\lambda_{2}+\lambda_{3}\right) v_{\Delta}^{2}
\end{array}
$$

The squared mass of the (dominantly triplet) CP-odd $A^{0}$ is given by:

$$
M_{A^{0}}^{2}=M_{\Delta}^{2}+\left(\frac{\lambda_{1}}{2}+\frac{\lambda_{4}}{2}\right) v^{2}+\left(\lambda_{2}+\lambda_{3}\right) v_{\Delta}^{2}
$$

The squared mass of the (dominantly triplet) $H^{ \pm}$is given by:

$$
M_{H^{ \pm}}^{2}=M_{\Delta}^{2}+\left(\frac{\lambda_{1}}{2}+\frac{\lambda_{4}}{4}\right) v^{2}+\left(\lambda_{2}+\sqrt{2} \lambda_{3}\right) v_{\Delta}^{2}
$$

Finally, the squared mass of the (purely triplet) doubly-charged scalar $\left(H^{ \pm \pm}=\delta^{ \pm \pm}\right)$is given by:

$$
M_{H^{ \pm \pm}}^{2}=M_{\Delta}^{2}+\frac{\lambda_{1}}{2} v^{2}+\lambda_{2} v_{\Delta}^{2}
$$

One can see that the squared mass of the (dominantly doublet) $H_{2}$ is simply given by $2 \lambda v^{2}$, as in the SM. In the expressions for the masses of $M_{A^{0}}^{2}, M_{H_{1}}^{2}, M_{H^{ \pm}}^{2}$ and $M_{H^{ \pm \pm}}^{2}$ there is a common term $M_{\Delta}^{2}+\frac{\lambda_{1}}{2} v^{2}$. It is evident that the mass scales for $H_{2}$ and the dominantly triplet scalars $\left(A^{0}, H_{1}, H^{ \pm}, H^{ \pm \pm}\right)$are unrelated, the former being set by $2 \lambda v^{2}$ and the latter by $M_{\Delta}^{2}+\frac{\lambda_{1}}{2} v^{2}$. Neglecting the terms which are proportional to the small parameter $v_{\Delta}$, one can see that there are only two possible mass hierarchies for the triplet scalars, with the magnitude of the mass splitting being controlled by $\lambda_{4}\left(\right.$ and $\left.M_{A^{0}}=M_{H_{1}}\right)$ :

$$
\begin{aligned}
& M_{A^{0}}, M_{H_{1}}<M_{H^{ \pm}}<M_{H^{ \pm \pm}} \text {for } \lambda_{4}<0 \\
& M_{H^{ \pm \pm}}<M_{H^{ \pm}}<M_{A^{0}}, M_{H_{1}} \text { for } \lambda_{4}>0
\end{aligned}
$$

\footnotetext{
${ }^{2}$ The mixing angle can be maximal in the region of degeneracy $M_{H_{2}} \sim M_{H_{1}}$, but it quickly becomes small $\left(\sim v_{\Delta} / v\right)$ with increasing mass splitting $\left|M_{H_{2}}-M_{H_{1}}\right| 31$ 33].
} 
In studies of the HTM it is sometimes assumed that $M_{\Delta}^{2} \gg 2 \lambda v^{2}$ i.e. $M_{H^{ \pm \pm}}, M_{H^{ \pm}}, M_{H_{1}}, M_{A^{0}} \gg M_{H_{2}}$. The motivation for this scenario is to have a "seesaw type" explanation for the smallness of $v_{\Delta}$ in eq. (5). However, for $M_{\Delta}$ much larger than the TeV scale there would be no hope of observing the triplet scalars at the LHC. In recent years there has been much interest in the study of the HTM as a TeV scale model of neutrino mass generation [10 14, 19 29] i.e. not invoking a large mass scale for $M_{\Delta}$. In these studies it is assumed (either explicitly or implicitly) that $M_{\Delta}^{2}>2 \lambda v^{2}$, with $M_{\Delta}<1 \mathrm{TeV}$.

The converse case where $M_{\Delta}^{2}+\frac{\lambda_{1}}{2} v^{2}<2 \lambda v^{2}$ is rarely considered. In [31 33] the possibility of $M_{H_{2}}>M_{H^{ \pm \pm}}, M_{H^{ \pm}}, M_{H_{1}}, M_{A^{0}}$ has been mentioned, and in [33] the case of $M_{H_{2}}>$ $2 M_{\phi}$ (where $\phi$ is one of $H^{ \pm \pm}, H^{ \pm}, H_{1}, A^{0}$ ) is explicitly discussed. However, in these works there is no study of the phenomenology of $H_{2}$ at hadron colliders for the case of $M_{H_{2}}>$ $2 M_{\phi}$, and how its experimental signature might differ from that of the SM Higgs Boson. Importantly, if $M_{H_{2}}>2 M_{\phi}$ then new decay channels for $H_{2}$ become possible, ${ }^{3}$ namely $H_{2} \rightarrow H^{++} H^{--}, H^{+} H^{-}, H_{1} H_{1}, A^{0} A^{0}$.

In this case the phenomenology of $H_{2}$ in the HTM could be different to that of the SM Higgs boson, because the new decay channels (if open kinematically) would compete with the usual decays of $\mathrm{H}_{2}$ to SM particles (i.e. $H_{2} \rightarrow W W, Z Z, t \bar{t}$ ). Of particular interest is the decay $H_{2} \rightarrow H^{++} H^{--}$, for which the condition $M_{H_{2}}>2 M_{H^{ \pm \pm}}$is necessary. If its branching ratio were sizeable then the production of $H_{2}$ via gluon-gluon fusion $g g \rightarrow H_{2}$ followed by the decay $\mathrm{H}_{2} \rightarrow \mathrm{H}^{++} \mathrm{H}^{--}$would be an additional way to produce a pair of $H^{ \pm \pm}$at hadron colliders.

We note that the condition $M_{H_{2}}>2 M_{H^{ \pm \pm}}$necessarily requires $M_{H_{2}} \gtrsim 200 \mathrm{GeV}$ in order to respect the current lower bounds on $M_{H^{ \pm \pm}}$from direct searches. At first sight, such a heavy SM-like $\mathrm{H}_{2}$ would appear to be in conflict with experimental data, since it is well known that the Higgs boson in the SM is expected to be lighter than $200 \mathrm{GeV}$ in order not to give an unacceptably large contribution to electroweak precision observables. In the context of the SM the case of $M_{H_{2}} \gtrsim 200 \mathrm{GeV}$ is quite strongly disfavoured, although this fact has not dissuaded direct searches in this mass region at the LHC [34, 35]. However, the bound $M_{H_{2}} \gtrsim 200 \mathrm{GeV}$ cannot strictly be applied to the HTM, due to the additional scalar particles and the different renormalisation procedure, the latter being necessary because of the presence of the triplet vev $\left(v_{\Delta}\right)$. Dedicated analyses in models with scalar triplets have shown that a heavy (up to $1 \mathrm{TeV}$ ) SM-like Higgs boson can be made consistent with electroweak precision measurements [40, 41]. These studies are for a model with a real $Y=0$ scalar triplet, which has no doubly charged scalar and gives $\rho>1$ at tree level, in contrast to the HTM which has $\rho<1$ at tree level, (eq. (66)). One can see in [41] that the condition $M_{H_{2}}>2 M_{\phi}$ (where $\phi$ is one of the $Y=0$ triplet scalars) can be be accommodated. Although there is no explicit study in the HTM, we expect this result to also hold due to its greater number of free parameters (i.e. particle masses). In our numerical analysis we will treat $M_{H_{2}}<700 \mathrm{GeV}$ and $M_{H_{2}}>2 M_{H^{ \pm \pm}}$as permissible parameter space in the HTM.

From a phenomenological point of view, a heavy $(>>200 \mathrm{GeV}$ ) SM-like Higgs boson is attractive because it would be discovered more quickly at the LHC than a light SM-like Higgs boson with mass $<140 \mathrm{GeV}$. The region of $200 \mathrm{GeV}<M_{H_{2}}<500 \mathrm{GeV}$, for which the decays $H_{2} \rightarrow Z Z$ and $H_{2} \rightarrow W W$ are dominant in the SM, is a mass range where the LHC has sensitivity to cross sections which are much smaller than that of the SM Higgs boson. The

\footnotetext{
${ }^{3}$ The scenario of a heavy SM-like Higgs boson decaying to singly charged scalars, $h^{0} \rightarrow H^{+} H^{-}$, has been discussed in the Two Higgs Doublet Model [42].
} 
first searches at the LHC for a SM Higgs with $M_{H_{2}}>200 \mathrm{GeV}$ have already been carried out. The ATLAS collaboration (with $36 \mathrm{pb}^{-1}$ of integrated luminosity) has searched for $H_{2} \rightarrow Z Z$ with the decay modes $Z Z \rightarrow \ell^{+} \ell^{-} \nu \nu, Z Z \rightarrow \ell^{+} \ell^{-} q \bar{q}$ and $Z Z \rightarrow \ell^{+} \ell^{-} \ell^{+} \ell^{-}$, as well as $H_{2} \rightarrow W W$ with the decay mode $W W \rightarrow \ell \nu q^{\prime} \bar{q}$ [34]. The CMS collboration has searched for $\mathrm{H}_{2} \rightarrow W W$ with the decay mode $W W \rightarrow \ell \nu \ell \nu$ [35]. Production of $\mathrm{H}_{2}$ is assumed to be via gluon-gluon fusion, $g g \rightarrow H_{2}$, and cross sections which are an order of magnitude above the prediction of the SM are currently being excluded at $95 \%$ c.l. By the end of the $\sqrt{s}=7$ $\mathrm{TeV}$ run (in which a few $\mathrm{fb}^{-1}$ of integrated luminosity will be accumulated), the sensitivity in these channels will be sufficient to exclude or provide evidence for the SM Higgs boson at a high confidence level in the region $200 \mathrm{GeV}<M_{H_{2}}<500 \mathrm{GeV}$. If the branching ratios of $H_{2} \rightarrow H^{++} H^{--}, H^{+} H^{-}, H_{1} H_{1}, A^{0} A^{0}$ were sizeable then discovery of $H_{2}$ in the channels $\mathrm{H}_{2} \rightarrow Z Z$ and $\mathrm{H}_{2} \rightarrow W W$ would require more integrated luminosity.

\section{THE DECAYS $H_{2} \rightarrow \phi \phi$ WITH $\phi=H^{ \pm \pm}, H^{ \pm}, H_{1}, A^{0}$}

There are four decay channels of $H_{2}$ to pairs of scalars in the HTM: $H_{2} \rightarrow H^{++} H^{--}$, $H_{2} \rightarrow H^{+} H^{-}, H_{2} \rightarrow A^{0} A^{0}$ and $H_{2} \rightarrow H_{1} H_{1}$. If $M_{H_{2}}>2 M_{\phi}\left(\right.$ where $\left.\phi=H^{ \pm \pm}, H^{ \pm}, H_{1}, A^{0}\right)$ one can treat this as a two-body decay to a pair of on-shell $\phi$. If $M_{H_{2}}<2 M_{\phi}$ we consider the partial width to be zero. Between one and four of the decays $H_{2} \rightarrow \phi \phi$ can be open kinematically, depending on the mass splitting among $\phi$ (which is controlled by $\lambda_{4}$ in eq. (13) and eq. (14). For $\lambda_{4}>0$ the lightest of the triplet scalars is $H^{ \pm \pm}$. In this scenario, once values of $M_{H_{2}}$ and $M_{H^{ \pm \pm}}$are chosen such that $M_{H_{2}}>2 M_{H^{ \pm \pm}}$there will be a value of $\lambda_{4}$ above which only $\mathrm{H}_{2} \rightarrow \mathrm{H}^{++} \mathrm{H}^{--}$is kinematically open. This will be the scenario where $\mathrm{BR}\left(\mathrm{H}_{2} \rightarrow \mathrm{H}^{++} \mathrm{H}^{--}\right)$is maximal.

The Feynman rules for the scalar trilinear couplings which mediate the decays are as follows (omitting a factor of $-i$ ):

$$
\begin{array}{r}
C_{H_{2} H^{++} H^{--}}=\lambda_{1} v \\
C_{H_{2} H^{+} H^{-}}=\left(\lambda_{1}+\frac{\lambda_{4}}{2}\right) v \\
C_{H_{2} H_{1} H_{1}}, C_{H_{2} A^{0} A^{0}}=\left(\lambda_{1}+\lambda_{4}\right) v
\end{array}
$$

Here we consider $H_{2}$ to be entirely composed of the isospin doublet scalar field, which is true to a very good approximation. One can see that $C_{H_{2} H^{++} H^{--}}$is controlled only by $\lambda_{1}$, while the other trilinear couplings depend on both $\lambda_{1}$ and $\lambda_{4}$. If $\lambda_{1}$ and $\lambda_{4}$ are sizeable, then the branching ratios for $H_{2} \rightarrow H^{++} H^{--}, H_{2} \rightarrow H^{+} H^{-}, H_{2} \rightarrow H_{1} H_{1}$, and $H_{2} \rightarrow A^{0} A^{0}$ could be non-negligible.

One can use a generic formula for the decay rate for the four channels:

$$
\Gamma\left(H_{2} \rightarrow \phi \phi\right)=\delta_{H} \frac{\left|C_{H_{2} \phi \phi}\right|^{2}}{32 \pi M_{H_{2}}}\left(1-\frac{4 M_{\phi}^{2}}{M_{H_{2}}^{2}}\right)^{1 / 2}
$$

Here $\delta_{H}=2$ for $\phi=H^{ \pm \pm}, H^{ \pm}$(i.e. non-identical particles in the final state) and $\delta=1$ for $\phi=H_{1}, A^{0}$ (i.e. identical particles in the final state)

It is clear that the two crucial parameters for a large $\operatorname{BR}\left(H_{2} \rightarrow H^{++} H^{--}\right)$are $\lambda_{1}$ (which determines the strength of the coupling $\left|C_{H_{2} H^{++} H^{--}}\right|$in eq. (15) and $M_{H^{ \pm \pm}}$(which determines the suppression from phase space). In our numerical analysis we shall take 
$M_{H^{ \pm \pm}}$as an input parameter. As can be seen from eq. (12), the dominant contribution to $M_{H^{ \pm \pm}}^{2}$ is from the two terms $M_{\Delta}^{2}+\lambda_{1} v^{2} / 2$. Therefore, by taking $\lambda_{1}$ and $M_{H^{ \pm \pm}}$as input parameters the value of $M_{\Delta}^{2}$ is determined. We will be focussing on the parameter space of $90 \mathrm{GeV}<M_{H^{ \pm \pm}}<300 \mathrm{GeV}$ and $0<\lambda_{1}<4$, and consequently $M_{\Delta}^{2}<0$ when $M_{H^{ \pm \pm}}^{2}<\lambda_{1} v^{2} / 2$. In the scenario of $M_{\Delta}^{2}<0$ the positive mass of $M_{H^{ \pm \pm}}$is obtained from the term $\lambda_{1} v^{2} / 2$. Alternatively, one could consider $\lambda_{1}<0$ and $M_{\Delta}^{2}>0$. The crucial point here is that the parameters $M_{\Delta}^{2}$ and $\lambda_{1}$ should have opposite signs if one wishes to have large $\left|\lambda_{1}\right|$ (in order to enhance $\left|C_{H_{2} H^{++} H^{--}}\right|$) together with a fairly light $H^{ \pm \pm}$.

We now summarise the current lower limits on $M_{H^{ \pm \pm}}$from direct searches. There have been searches for the decay channels $H^{ \pm \pm} \rightarrow \ell_{i}^{ \pm} \ell_{j}^{ \pm}$for $i, j=e, \mu, \tau$ (these are the dominant decay channels for $v_{\Delta} \lesssim 0.1 \mathrm{MeV}$ ) at LEP [43], Tevatron [15-18] and the LHC [30] (CMS Collaboration). The strongest mass limits for the decays $H^{ \pm \pm} \rightarrow e e, e \mu, \mu \mu$ are from the LHC search, which obtained $M_{H^{ \pm \pm}}>144,154,156 \mathrm{GeV}$ respectively, assuming $\mathrm{BR}=100 \%$ in a given channel. Separate searches for three and four leptons (which have significantly different backgrounds) were performed. These limits are weakened considerably for the case of $\mathrm{BR}<100 \%$ because the event number for the signal is proportional to the square of the branching ratio $\left(\mathrm{BR}^{2}\right)$. In [30] both production mechanisms $q \bar{q} \rightarrow \gamma^{*}, Z^{*} \rightarrow H^{++} H^{--}$ and $q^{\prime} \bar{q} \rightarrow W \rightarrow H^{ \pm \pm} H^{\mp}$ were considered (with the assumption $M_{H^{ \pm \pm}}=M_{H^{ \pm}}$), which increases the sensitivity in the three-lepton channel. For the decays involving one $\tau$, namely $H^{ \pm \pm} \rightarrow e \tau, \mu \tau$, the limit $M_{H^{ \pm \pm}}>106 \mathrm{GeV}$ was derived in both channels at the LHC [30], with stronger limits from the Tevatron $\left(M_{H^{ \pm \pm}}>112 \mathrm{GeV}\right.$ and $114 \mathrm{GeV}$ respectively) obtained in [18]. The only search for $H^{ \pm \pm} \rightarrow \tau^{ \pm} \tau^{ \pm}$at a hadron collider is the LHC search in [30], which derived the limit $M_{H^{ \pm \pm}} \gtrsim 80 \mathrm{GeV}$.

We will take $M_{H^{ \pm \pm}}=90 \mathrm{GeV}$ as our lowest value for the mass of $H^{ \pm \pm}$, and this is allowed for certain choices of branching ratios of $H^{ \pm \pm}$. As explained above, the limits on $M_{H^{ \pm \pm}}$from hadron colliders are weakest for those channels which involve $\tau$. In contrast, the limit from the LEP searches of $M_{H^{ \pm \pm}} \gtrsim 100 \mathrm{GeV}$ applies to all the decays $H^{ \pm \pm} \rightarrow \ell_{i}^{ \pm} \ell_{j}^{ \pm}$with $i, j=e, \mu, \tau$. The search strategy at LEP requires four leptons and so the event number for the signal is proportional to $\mathrm{BR}^{2}$. The scenario of $M_{H^{ \pm \pm}}=90 \mathrm{GeV}$ is compatible with the all the above searches provided that the decays involving $\tau$ are dominant e.g. choices like $\mathrm{BR}\left(H^{ \pm \pm} \rightarrow e \tau, \mu \tau, \tau \tau\right)$ of around $33 \%$. It is not necessary to have $\mathrm{BR}\left(H^{ \pm \pm} \rightarrow e e, e \mu, \mu \mu\right)$ totally absent for $M_{H^{ \pm \pm}}=90 \mathrm{GeV}$, and BRs of the order of $10 \%$ for these channels can be accommodated because the event number is proportional to $\mathrm{BR}^{2}$, and for $\mathrm{BR}=10 \%$ this is a large suppression factor. We note that the sum of $\mathrm{BR}\left(H^{ \pm \pm} \rightarrow e e, e \mu, \mu \mu\right)$ cannot be taken arbitrarily small in the HTM because the Yukawa couplings $h_{i j}$ are related to the neutrino mass matrix via eq. (3)). The allowed values of $\mathrm{BR}\left(H^{ \pm \pm} \rightarrow \ell_{i}^{ \pm} \ell_{j}^{ \pm}\right)$in the HTM have been studied in detail in $[21$ 26], and in [25] it can be seen explicitly that the sum of $\mathrm{BR}\left(H^{ \pm \pm} \rightarrow e e, e \mu, \mu \mu\right)$ must be greater than around $5 \%$.

Very recently the searches for $H^{ \pm \pm} \rightarrow e e, e \mu, \mu \mu$ by the CDF collaboration in [15] (which used $0.24 \mathrm{fb}^{-1}$ ) were updated using $6.1 \mathrm{fb}^{-1}$ [44]. Mass limits of $M_{H^{ \pm \pm}}>225,210,245 \mathrm{GeV}$ were obtained, again assuming $\mathrm{BR}=100 \%$. In these searches the event number for the signal is linear in $\mathrm{BR}$, and for $\mathrm{BR} \sim 3 \%(15 \%)$ the limit $M_{H^{ \pm \pm}}>245 \mathrm{GeV}$ for $H^{ \pm \pm} \rightarrow \mu \mu$ would weaken to $M_{H^{ \pm \pm}}>100 \mathrm{GeV}(150 \mathrm{GeV})$. Note that these mass limits in [44] only assume production of $H^{ \pm \pm}$from $q \bar{q} \rightarrow \gamma^{*}, Z^{*} \rightarrow H^{++} H^{--}$. The inclusion of $q^{\prime} \bar{q} \rightarrow W \rightarrow H^{ \pm \pm} H^{\mp}$ would allow larger values of $M_{H^{ \pm \pm}}$to be probed. Finally, if the decay $H^{ \pm \pm} \rightarrow W^{ \pm} W^{ \pm}$is dominant (which is the case for $v_{\Delta} \gtrsim 0.1 \mathrm{MeV}$ ) then $M_{H^{ \pm \pm}}=90 \mathrm{GeV}$ is permitted because there have been no direct searches for this channel in the context of models with $H^{ \pm \pm}$. We 
will respect the all the above mass limits in our numerical analysis, the most stringent ones being for the channels $H^{ \pm \pm} \rightarrow e^{ \pm} e^{ \pm}, e^{ \pm} \mu^{ \pm}$and $\mu^{ \pm} \mu^{ \pm}$.

We will only consider the scenario of $M_{H_{2}}>200 \mathrm{GeV}$ for which the decay channels $\mathrm{H}_{2} \rightarrow W W$ and $\mathrm{H}_{2} \rightarrow Z Z$ can be treated as two-body decays. The expressions for their partial decay widths are as follows:

$$
\Gamma\left(H_{2} \rightarrow W W\right)=\frac{\sqrt{2} G_{F} M_{H_{2}}^{3}}{32 \pi}\left(1-4 \kappa_{W}+12 \kappa_{W}^{2}\right)\left(1-4 \kappa_{W}\right)^{1 / 2} \delta_{W}
$$

with $\kappa_{W}=M_{W}^{2} / M_{H_{2}}^{2}$ and $\delta_{W}=2$.

$$
\Gamma\left(H_{2} \rightarrow Z Z\right)=\frac{\sqrt{2} G_{F} M_{H_{2}}^{3}}{32 \pi}\left(1-4 \kappa_{Z}+12 \kappa_{Z}^{2}\right)\left(1-4 \kappa_{Z}\right)^{1 / 2} \delta_{Z}
$$

with $\kappa_{Z}=M_{Z}^{2} / M_{H_{2}}^{2}$ and $\delta_{Z}=1$. If $M_{H_{2}}>2 m_{t}$ then the decay channel $H_{2} \rightarrow t \bar{t}$ is open:

$$
\Gamma(H \rightarrow \bar{t} t)=\frac{3 G_{F} m_{t}^{2}}{4 \sqrt{2} \pi} M_{H} \beta_{t}^{3}
$$

where $\beta_{t}=\left(1-4 m_{t}^{2} / M_{H_{2}}^{2}\right)^{1 / 2}$. All other decays of $H_{2}$ to SM particles (e.g. $\left.H_{2} \rightarrow b \bar{b}, \tau^{+} \tau^{-}\right)$ have negligibly small partial widths for $M_{H_{2}}>200 \mathrm{GeV}$. Note that other decay channels such as $H_{2} \rightarrow H^{ \pm} W, H_{2} \rightarrow H_{1} W$ and $H_{2} \rightarrow A^{0} Z$ are suppressed by the small mixing between the doublet and triplet fields, and so can be neglected.

\section{NUMERICAL ANALYSIS}

We now study the magnitude of the branching ratios of the decays channels $H_{2} \rightarrow \phi \phi$ for $\phi=H^{ \pm \pm}, H^{ \pm}, H_{1}, A^{0}$. The four important parameters are $M_{H^{ \pm \pm}}, M_{H_{2}}, \lambda_{1}$ and $\lambda_{4}$. The other parameters in the scalar potential are fixed as $\lambda_{2}=\lambda_{3}=0.5$ and $v_{\Delta}=10^{-2}$ $\mathrm{MeV}$, the latter choice ensuring that the decays $H^{ \pm \pm} \rightarrow \ell^{ \pm} \ell^{ \pm}$are dominant. These latter three parameters appear in the expressions for the masses of the triplet scalars in eq. (9) to eq. (12) but their effect is essentially negligible, even for the case of $v_{\Delta}=1 \mathrm{GeV}$. We will present results for $M_{H^{ \pm \pm}}=90 \mathrm{GeV}, 150 \mathrm{GeV}, 200 \mathrm{GeV}$ and $300 \mathrm{GeV}$. As explained in the previous section, the choice of $M_{H^{ \pm \pm}}=90 \mathrm{GeV}$ requires small BRs $(<3 \%)$ for the decay channels $H^{ \pm \pm} \rightarrow e^{ \pm} e^{ \pm}, e^{ \pm} \mu^{ \pm}$and $\mu^{ \pm} \mu^{ \pm}$(and consequently large BRs to channels involving $\tau)$ in order to respect the limits from the direct searches for $H^{ \pm \pm}$. Larger values $(\gg 3 \%)$ of $\mathrm{BR}\left(H^{ \pm \pm} \rightarrow e^{ \pm} e^{ \pm}, e^{ \pm} \mu^{ \pm}, \mu^{ \pm} \mu^{ \pm}\right)$are permitted as $M_{H^{ \pm \pm}}$increases.

Fig. (17a) shows the branching ratios of $H_{2}$ as a function of $M_{H_{2}}$. We take $M_{H^{ \pm \pm}}=90$ $\mathrm{GeV}, \lambda=1$, and $\lambda_{4}=0.8$ (the latter choice gives $M_{H^{ \pm}}=142 \mathrm{GeV}$ and $M_{A^{0}, H^{0}}=179 \mathrm{GeV}$ ). The magnitude of $\mathrm{BR}\left(H_{2} \rightarrow H^{++} H^{--}\right)$can reach $65 \%$ for $M_{H_{2}}=200 \mathrm{GeV}$, and stays as the dominant channel until $M_{H_{2}} \sim 260 \mathrm{GeV}$, at which $H_{2} \rightarrow W W$ becomes dominant. $\mathrm{BR}\left(H_{2} \rightarrow\right.$ $\left.H^{++} H^{--}\right)$falls below $\mathrm{BR}\left(H_{2} \rightarrow Z Z\right)$ at around $M_{H_{2}}=320 \mathrm{GeV}$. This dependence on $M_{H_{2}}$ can be explained by the fact that the partial widths of $H_{2} \rightarrow W W, Z Z$ are proportional to $M_{H_{2}}^{3}$, and so ultimately these channels will dominate for larger $M_{H_{2}}$. The other decays of $\mathrm{H}_{2}$ to two triplet scalars also can have sizeable branching ratios, with $\mathrm{BR}\left(\mathrm{H}_{2} \rightarrow \mathrm{H}^{+} \mathrm{H}^{-}\right)$ reaching $20 \%$ at most, and exceeds $\mathrm{BR}\left(H_{2} \rightarrow H^{++} H^{--}\right)$for $M_{H_{2}} \gtrsim 315 \mathrm{GeV}$. The branching ratios of $H_{2} \rightarrow A^{0} A^{0}$ and $H_{2} \rightarrow H_{1} H_{1}$ are equal; they are plotted individually and their sum peaks at $\sim 10 \%$. In fig. (1 $\mathrm{b})$ we show contours of $\mathrm{BR}\left(H_{2} \rightarrow H^{++} H^{--}\right)$in the plane $\left[M_{H_{2}}, \lambda_{1}\right]$. 
As expected, $\mathrm{BR}\left(H_{2} \rightarrow H^{++} H^{--}\right)$takes its largest values for large $\lambda_{1}$ and light $M_{H_{2}}$, with $\mathrm{BR}\left(\mathrm{H}_{2} \rightarrow \mathrm{H}^{++} \mathrm{H}^{--}\right)>90 \%$ being possible. We note that such a scenario would render the searches for $\mathrm{H}_{2} \rightarrow W W, Z Z$ ineffective until a very large amount of integrated luminosity is obtained. In fig. (11c) we show contours of $\mathrm{BR}\left(H_{2} \rightarrow H^{++} H^{--}\right)$in the plane $\left[\lambda_{4}, \lambda_{1}\right]$, fixing $M_{H_{2}}=300 \mathrm{GeV}$. For $\lambda_{4} \gtrsim 1$ only the decay $H_{2} \rightarrow H^{++} H^{--}$is open kinematically and so the contours are horizontal. For $\lambda_{4}=0$ all the triplet scalars are degenerate and thus all four decay channels are open. Figs. 2, 3] and 4 are analogies of fig. 1, but with $M_{H^{ \pm \pm}}=150$ $\mathrm{GeV}, 200 \mathrm{GeV}$ and $300 \mathrm{GeV}$ respectively. In all figures we take $M_{H_{2}}>2 M_{H^{ \pm \pm}}$. One can see similar qualitative behaviour, but since the lowest value of $M_{H_{2}}$ is larger in figs. 2, 3 and 4 than in fig. 1, the maximum values of $\mathrm{BR}\left(H_{2} \rightarrow H^{++} H^{--}\right)$are less than in fig.1. However, in fig. 2b, fig. $3 \mathrm{~b}$ and fig. $4 \mathrm{~b}$ it can be seen that $\operatorname{BR}\left(H_{2} \rightarrow H^{++} H^{--}\right)>50 \%, 25 \%, 5 \%$ respectively is possible for $\lambda_{1} \gtrsim 3$.

It clear that $\mathrm{BR}\left(\mathrm{H}_{2} \rightarrow \mathrm{H}^{++} \mathrm{H}^{--}\right)$can be sizeable, and we will now quantify the magnitude of the pair production of $H^{ \pm \pm}$which originates from production and decay of $H_{2}$. At hadron colliders $H_{2}$ is dominantly created via gluon-gluon fusion, $g g \rightarrow H_{2}$. For $M_{H_{2}}=2 M_{H^{ \pm \pm}}$the cross section of $g g \rightarrow H_{2}$ at the LHC is significantly larger than the cross section for the direct production mechanisms of $H^{ \pm \pm}$(i.e. $q \bar{q} \rightarrow \gamma^{*}, Z^{*} \rightarrow H^{++} H^{--}$ and $\left.q^{\prime} \bar{q} \rightarrow W \rightarrow H^{ \pm \pm} H^{\mp}\right)$. However, the same is not true at the Tevatron, and $\sigma\left(g g \rightarrow H_{2}\right) \lesssim \sigma\left(q \bar{q} \rightarrow \gamma^{*}, Z^{*} \rightarrow H^{++} H^{--}\right)$for $M_{H_{2}}>2 M_{H^{ \pm \pm}}$.

We introduce the ratio $R$, defined by:

$$
R=\frac{\sigma\left(g g \rightarrow H_{2}\right) \times \mathrm{BR}\left(H_{2} \rightarrow H^{++} H^{--}\right)}{\sigma\left(q \bar{q} \rightarrow \gamma^{*}, Z^{*} \rightarrow H^{++} H^{--}\right)}
$$

The denominator in eq. (22) is the conventional mechanism for production of $\mathrm{H}^{++} \mathrm{H}^{--}$, which is assumed in the ongoing searches for $H^{ \pm \pm}$. The numerator is a novel mechanism which contributes when $\operatorname{BR}\left(H_{2} \rightarrow H^{++} H^{--}\right) \neq 0$. We will now study the magnitude of the ratio $R$ at the LHC (with $\sqrt{s}=7 \mathrm{TeV}$ and $14 \mathrm{TeV}$ ) and at the Tevatron. In Fig. 5 we plot $R$ as a function of $M_{H_{2}}$ at the LHC with $\sqrt{s}=14 \mathrm{TeV}$, for $M_{H^{ \pm \pm}}=90 \mathrm{GeV}, 150 \mathrm{GeV}$, $200 \mathrm{GeV}$ and $300 \mathrm{GeV}$. The factorisation scale and normalisation scale are both taken to be $M_{H_{2}}$ for $g g \rightarrow H_{2}$, while for $q \bar{q} \rightarrow \gamma^{*}, Z^{*} \rightarrow H^{++} H^{--}$both scales are taken to be the partonic centre-of-mass energy. We use CTEQ6L1 parton distribution functions [45] with the leading-order partonic cross section for $g g \rightarrow H_{2}[46]$. We do not apply QCD $K$ factors which, would increase the value of $R$ because the ratio of the $K$ factors for $\sigma\left(g g \rightarrow H_{2}\right)$ [47] and $\sigma\left(q \bar{q} \rightarrow \gamma^{*}, Z^{*} \rightarrow H^{++} H^{--}\right)$[10] is about 1.4 in the region of interest of $M_{H_{2}}$ and $M_{H \pm \pm}$.

In fig. (5a) we take $M_{H^{ \pm \pm}}=90 \mathrm{GeV}$, which fixes the value of $\sigma\left(q \bar{q} \rightarrow H^{++} H^{--}\right)$, and $\lambda_{4}=0.8$. We take $\lambda_{1}=1$ and 4 . If $\lambda_{1}=1$ one can see that $R=4.7$ for $M_{H_{2}}=200 \mathrm{GeV}$, and $R>1$ for $M_{H_{2}}<290 \mathrm{GeV}$. If $\lambda_{1}=4$, one finds that $R=7.0$ for $M_{H_{2}}=200 \mathrm{GeV}$, and $R>1$ for $M_{H_{2}}<420 \mathrm{GeV}$. The noticeable drop in the value of $R$ for $M_{H_{2}} \sim 280 \mathrm{GeV}$ is due to the opening of the decay channel $H_{2} \rightarrow H^{+} H^{-}$(see fig. (1a)). Both $\sigma\left(g g \rightarrow H_{2}\right.$ ) and $\mathrm{BR}\left(\mathrm{H}_{2} \rightarrow \mathrm{H}^{++} \mathrm{H}^{--}\right)$are decreasing functions of $M_{H_{2}}$, which explains the overall dependence of $R$ on $M_{H_{2}}$. Note that $R$ does not fall so sharply with $M_{H_{2}}$ in the region $320 \mathrm{GeV}<$ $M_{H_{2}}<380 \mathrm{GeV}$, because $\sigma\left(g g \rightarrow H_{2}\right)$ increases in magnitude up to a local maximum at $M_{H_{2}}=2 m_{t}$, before decreasing again. In fig. (5b) we take $M_{H^{ \pm \pm}}=150 \mathrm{GeV}$, and $R \sim 16$ for $M_{H_{2}}=2 m_{t}$ and $\lambda_{1}=4$. Larger values of $R$ are attainable because the magnitude of $\sigma\left(q \bar{q} \rightarrow \gamma^{*}, Z^{*} \rightarrow H^{++} H^{--}\right)$(i.e. the denominator eq. (22) ) diminishes considerably when going from $M_{H^{ \pm \pm}}=90 \mathrm{GeV}$ to $M_{H^{ \pm \pm}}=150 \mathrm{GeV}$, while the corresponding decrease 
in $\sigma\left(g g \rightarrow H_{2}\right)$ for larger $M_{H_{2}}$ is relatively less. In fig. (5) (for $M_{H^{ \pm \pm}}=200 \mathrm{GeV}$ ) the maximum value is $R \sim 19$, and in fig. (5 $\mathrm{d})$ the maximum value is $R \sim 4$. It is evident that there is a sizeable parameter space for $R>1$, and thus $g g \rightarrow H_{2}$ could give a significant (or even dominant) contribution to the pair production of $H^{ \pm \pm}$at the LHC. We also note that the decay $\mathrm{H}_{2} \rightarrow \mathrm{H}^{+} \mathrm{H}^{-}$(which can have a large BR in fig. (1 $\mathrm{k}$ ) $\rightarrow$ fig. (4a)) can lead to additional production of $H^{++} H^{--}$because the branching ratio of the decay $H^{ \pm} \rightarrow H^{ \pm \pm} W^{*}$ can be large in a sizeable parameter space of $\left[v_{\Delta}, M_{H^{ \pm}}-M_{H^{ \pm \pm}}\right]$, as shown in [48]. In fact, in fig .(17a) $\rightarrow$ fig. (4ha) the mass splitting $\left(M_{H^{ \pm}}-M_{H^{ \pm \pm}}\right)$is between $20 \mathrm{GeV}$ and $52 \mathrm{GeV}$, and with our chosen value of $v_{\Delta}=10^{-2} \mathrm{MeV}$ one would have $\operatorname{BR}\left(H^{ \pm} \rightarrow H^{ \pm \pm} W^{*}\right)>99 \%$.

In fig. (6) we plot the analogies of fig. (5) for the LHC with $\sqrt{s}=7 \mathrm{TeV}$. One sees a similar qualitative behaviour, with lower maximum values of $R$. In fig. (7) we plot the corresponding results for the Tevatron, for $M_{H^{ \pm \pm}}=90 \mathrm{GeV}$ and $M_{H^{ \pm \pm}}=150 \mathrm{GeV}$. Since $\sigma\left(g g \rightarrow H_{2}\right) \lesssim \sigma\left(q \bar{q} \rightarrow \gamma^{*}, Z^{*} \rightarrow H^{++} H^{--}\right)$for $M_{H_{2}}>2 M_{H^{ \pm \pm}}$at the Tevatron, the maximum value of $R \sim 0.4$ (for $\lambda_{1}=4$ ) is much smaller than at the LHC and is comparable to the QCD K factor for $\sigma\left(q \bar{q} \rightarrow \gamma^{*}, Z^{*} \rightarrow H^{++} H^{--}\right)$[10].

Finally, we quantify the number of $H^{++} H^{--}$events for a given integrated luminosity $\mathcal{L}$ at the LHC. We introduce the parameter $N_{H^{ \pm \pm}}$, which is defined as follows:

$$
N_{H^{ \pm \pm}}=\epsilon \times \mathcal{L} \times\left[\sigma\left(q \bar{q} \rightarrow \gamma^{*}, Z^{*} \rightarrow H^{++} H^{--}\right)+\sigma\left(g g \rightarrow H_{2}\right) \times \mathrm{BR}\left(H_{2} \rightarrow H^{++} H^{--}\right)\right]
$$

The efficiency $\epsilon$ is the fraction of $H^{++} H^{--}$events which remain after all acceptance/selection cuts are imposed to reduce the background from the SM. The value of $\epsilon$ depends on which decay channel $H^{ \pm \pm} \rightarrow \ell_{i}^{ \pm} \ell_{j}^{ \pm}$is being considered. From the LHC simulation in [28] for the decay $H^{ \pm \pm} \rightarrow \mu^{ \pm} \mu^{ \pm}$with $\sqrt{s}=14 \mathrm{TeV}$, one can derive an approximate value of $\epsilon_{\mu \mu}=0.73$ for $M_{H^{ \pm \pm}}=600 \mathrm{GeV}$ and $\epsilon_{\mu \mu}=0.64$ for $M_{H^{ \pm \pm}}=300 \mathrm{GeV}$. As expected, the efficiency is greater for larger $M_{H^{ \pm \pm}}$, since the leptons originating from $H^{ \pm \pm}$are more energetic. Extrapolating these values to the region of $M_{H^{ \pm \pm}}<300 \mathrm{GeV}$ (the mass region on which we will focus) one roughly obtains $0.5<\epsilon_{\mu \mu}<0.6$. The efficiencies for the decay channels $H^{ \pm \pm} \rightarrow e^{ \pm} e^{ \pm}$and $H^{ \pm \pm} \rightarrow e^{ \pm} \mu^{ \pm}$are expected to be similar in magnitude to $\epsilon_{\mu \mu}$ (see [30]). The efficiencies for the decays of $H^{ \pm \pm}$to final states involving a $\tau$ lepton are much lower e.g. in [30] one can derive $\epsilon_{\mu \tau} \sim 0.02$ for the channel $H^{ \pm \pm} \rightarrow \mu^{ \pm} \tau^{ \pm}$, with even lower values for the channel $H^{ \pm \pm} \rightarrow \tau^{ \pm} \tau^{ \pm}$. We will show results for the decay mode $H^{ \pm \pm} \rightarrow \mu^{ \pm} \mu^{ \pm}$, for $M_{H^{ \pm \pm}}=90 \mathrm{GeV}, 150 \mathrm{GeV}, 200 \mathrm{GeV}$ and $300 \mathrm{GeV}$. The strongest lower bounds on $M_{H^{ \pm \pm}}$in this channel (assuming a branching ratio of $100 \%$ ) are $M_{H^{ \pm \pm}}>156 \mathrm{GeV}$ from the LHC in [30] and $M_{H^{ \pm \pm}}>245 \mathrm{GeV}$ from the Tevatron in [44], both limits being preliminary results. For the case of $\operatorname{BR}\left(H^{ \pm \pm} \rightarrow \mu^{ \pm} \mu^{ \pm}\right)<100 \%$, one can derive from [44] the approximate limits $M_{H^{ \pm \pm}} \gtrsim 100 \mathrm{GeV}, \gtrsim 150 \mathrm{GeV}$ and $\gtrsim 200 \mathrm{GeV}$ for $\mathrm{BR} \gtrsim 3 \%, \gtrsim 15 \%$ and $\gtrsim 40 \%$, respectively. We do not include these values of $\operatorname{BR}\left(H^{ \pm \pm} \rightarrow \mu^{ \pm} \mu^{ \pm}\right)$when showing results for $N_{H^{ \pm \pm}}$. In future searches which require three or four leptons (as done in the LHC search in [30]) the event number $N_{H^{ \pm \pm}}$in eq. (23) needs to be scaled by a multiplicative factor of $\left[\operatorname{BR}\left(H^{ \pm \pm} \rightarrow \ell_{i}^{ \pm} \ell_{j}^{ \pm}\right)\right]^{2}$. As explained above, this factor is necessarily less than unity if one considers $M_{H^{ \pm \pm}}<245 \mathrm{GeV}$ with decay $H^{ \pm \pm} \rightarrow \mu^{ \pm} \mu^{ \pm}$.

In fig. (88) we show $N_{H^{ \pm \pm}}$in the plane $\left[M_{H_{2}}, \lambda_{1}\right]$ for $\sqrt{s}=14 \mathrm{TeV}$ with $\mathcal{L}=30 \mathrm{fb}^{-1}$. We use $\epsilon_{\mu \mu}=0.64$ for $M_{H^{ \pm \pm}}=300 \mathrm{GeV}$, and $\epsilon_{\mu \mu}=0.50$ for the other chosen values of $M_{H^{ \pm \pm}}$ $(90 \mathrm{GeV}, 150 \mathrm{GeV}, 200 \mathrm{GeV})$. The contribution to $N_{H^{ \pm \pm}}$from $q \bar{q} \rightarrow \gamma^{*}, Z^{*} \rightarrow H^{++} H^{--}$ alone does not depend on $M_{H_{2}}$ and $\lambda_{1}$, and is roughly equal to 20500, 3270, 1130 and 280 for $M_{H^{ \pm \pm}}=90 \mathrm{GeV}, 150 \mathrm{GeV}, 200 \mathrm{GeV}$ and $300 \mathrm{GeV}$ respectively. In each panel in fig. (8) the contour with the lowest number of events corresponds to a value of $N_{H^{ \pm \pm}}$which is slightly 
larger than the above values for $N_{H^{ \pm \pm}}$from $q \bar{q} \rightarrow \gamma^{*}, Z^{*} \rightarrow H^{++} H^{--}$alone. We emphasise that the displayed $N_{H^{ \pm \pm}}$for $M_{H^{ \pm \pm}}=90 \mathrm{GeV}, 150 \mathrm{GeV}$ and $200 \mathrm{GeV}$ need to be multiplied by the square of BR (for a future three or four lepton search) where BR $\lesssim 3 \%, \lesssim 15 \%$ and $\lesssim 40 \%$ in order to comply with the mass limits in [44]. Fig. (8) can also be applied to other decay channels such as $H^{ \pm \pm} \rightarrow \mu^{ \pm} \tau^{ \pm}$after multiplying $N_{H^{ \pm \pm}}$by $\epsilon_{\mu \tau} / \epsilon_{\mu \mu} \sim 1 / 30$. Clearly, the contribution from $\sigma\left(g g \rightarrow H_{2}\right) \times \mathrm{BR}\left(H_{2} \rightarrow H^{++} H^{--}\right)$could significantly enhance the number of $H^{++} H^{--}$events at the LHC, provided that $M_{H_{2}}>2 M_{H^{ \pm \pm}}$and $\lambda_{1}$ is not very small. Since it is not expected that $M_{H_{2}} \gtrsim 700 \mathrm{GeV}$ (from considering constraints from perturbativity and unitarity e.g. see [33] $)$, the enhancement from $\sigma\left(g g \rightarrow H_{2}\right) \times \mathrm{BR}\left(H_{2} \rightarrow\right.$ $\left.H^{++} H^{--}\right)$is limited to the region $M_{H^{ \pm \pm}} \lesssim 350 \mathrm{GeV}$. However, its contribution would allow the possibility of probing smaller values of $\mathrm{BR}\left(H^{ \pm \pm} \rightarrow \ell_{i}^{ \pm} \ell_{j}^{ \pm}\right)$for a given value of $M_{H^{ \pm \pm}}$ (provided that $M_{H_{2}}>2 M_{H^{ \pm \pm}}$).

In fig. (9) we show $N_{H^{ \pm \pm}}$for $\sqrt{s}=7 \mathrm{TeV}$ with $\mathcal{L}=2 \mathrm{fb}^{-1}$. We take a slightly lower efficiency of $\epsilon_{\mu \mu}=0.4$ for $M_{H^{ \pm \pm}}=90 \mathrm{GeV}, 150 \mathrm{GeV}$ and $200 \mathrm{GeV}$, which is in rough agreement with the value for the channel $H^{ \pm \pm} \rightarrow \mu^{ \pm} \mu^{ \pm}$in the experimental search at $\sqrt{s}=7 \mathrm{TeV}$ in [30]. For $M_{H^{ \pm \pm}}=300 \mathrm{GeV}$ we take $\epsilon_{\mu \mu}=0.5$. Again, the enhancement from $\sigma\left(g g \rightarrow H_{2}\right) \times \mathrm{BR}\left(H_{2} \rightarrow H^{++} H^{--}\right)$can be sizeable, and could lead to a quicker discovery of a light $H^{ \pm \pm}$with $\operatorname{BR}\left(H^{ \pm \pm} \rightarrow \ell_{i}^{ \pm} \ell_{j}^{ \pm}\right)<100 \%$. We do not plot an analogous figure for the Tevatron since the maximum value of $R$ is much smaller than at the LHC, as shown in fig. (66).

Finally, we emphasise that the parameter space of $M_{H_{2}}>2 M_{H^{ \pm \pm}}$will be probed by two distinct searches with the LHC data taken at $\sqrt{s}=7 \mathrm{TeV}:$ i) the search for $H_{2} \rightarrow W W, Z Z$ (with first results in [34, 35]), and ii) the search for $q \bar{q} \rightarrow \gamma^{*}, Z^{*} \rightarrow H^{++} H^{--}$(with first results in [30], and a simulation in [49]). The run at $\sqrt{s}=7 \mathrm{TeV}$, with possibly up to 5 $\mathrm{fb}^{-1}$ of integrated luminosity, has the potential to exclude or provide evidence for a SM-like Higgs boson with $200 \mathrm{GeV}<M_{H_{2}}<500 \mathrm{GeV}$ at a high confidence level. Therefore the scenario of $M_{H_{2}}>2 M_{H^{ \pm \pm}}$and its possible impact on the direct searches for $H^{ \pm \pm}$should be clarified within the next two years.

\section{CONCLUSIONS}

Doubly charged Higgs bosons $\left(H^{ \pm \pm}\right)$, which arise in the Higgs Triplet Model (HTM) of neutrino mass generation, are being searched for at the Tevatron and at the LHC. The ongoing searches assume the production mechanisms $q \bar{q} \rightarrow \gamma^{*}, Z^{*} \rightarrow H^{++} H^{--}$and $q^{\prime} \bar{q} \rightarrow W^{*} \rightarrow H^{ \pm \pm} H^{\mp}$, with the leptonic decay $H^{ \pm \pm} \rightarrow \ell_{i}^{ \pm} \ell_{j}^{ \pm}$. We proposed an additional production mechanism for $H^{ \pm \pm}$, which becomes possible if the SM-like Higgs boson in the $\operatorname{HTM}\left(H_{2}\right)$ is heavy enough to decay to a pair of on-shell $H^{ \pm \pm}$. We quantified the magnitude of the branching ratio of $H_{2} \rightarrow H^{++} H^{--}$, and showed that it can be large $(>>10 \%)$ if a quartic coupling in the scalar potential is sizeable, $\lambda_{1}>1$. We performed a numerical study of the production rate for $\mathrm{H}_{2}$ via gluon-gluon fusion, $g \mathrm{~g} \rightarrow \mathrm{H}_{2}$, followed by the decay $\mathrm{H}_{2} \rightarrow \mathrm{H}^{++} \mathrm{H}^{--}$, and we showed that its cross section at the LHC can be greater than that of $q \bar{q} \rightarrow \gamma^{*}, Z^{*} \rightarrow H^{++} H^{--}$in a sizeable parameter space of $\left[\lambda_{1}, M_{H_{2}}\right]$ (with $M_{H_{2}}>2 M_{H^{ \pm \pm}}$). In the optimal case (e.g. $\lambda_{1}=4, M_{H_{2}} \sim 420 \mathrm{GeV}$ and $M_{H^{ \pm \pm}}=200 \mathrm{GeV}$ ) the ratio of cross sections can be as large as nineteen. We note that our analysis was carried out using the leading-order cross sections only, and the inclusion of QCD $K$ factors would provide a further enhancement of $40 \%$ in the above ratio. Such an additional source of $\mathrm{H}^{++} \mathrm{H}^{--}$ would enable smaller values of the branching ratio of $H^{ \pm \pm} \rightarrow \ell_{i}^{ \pm} \ell_{j}^{ \pm}$to be probed at the LHC. 

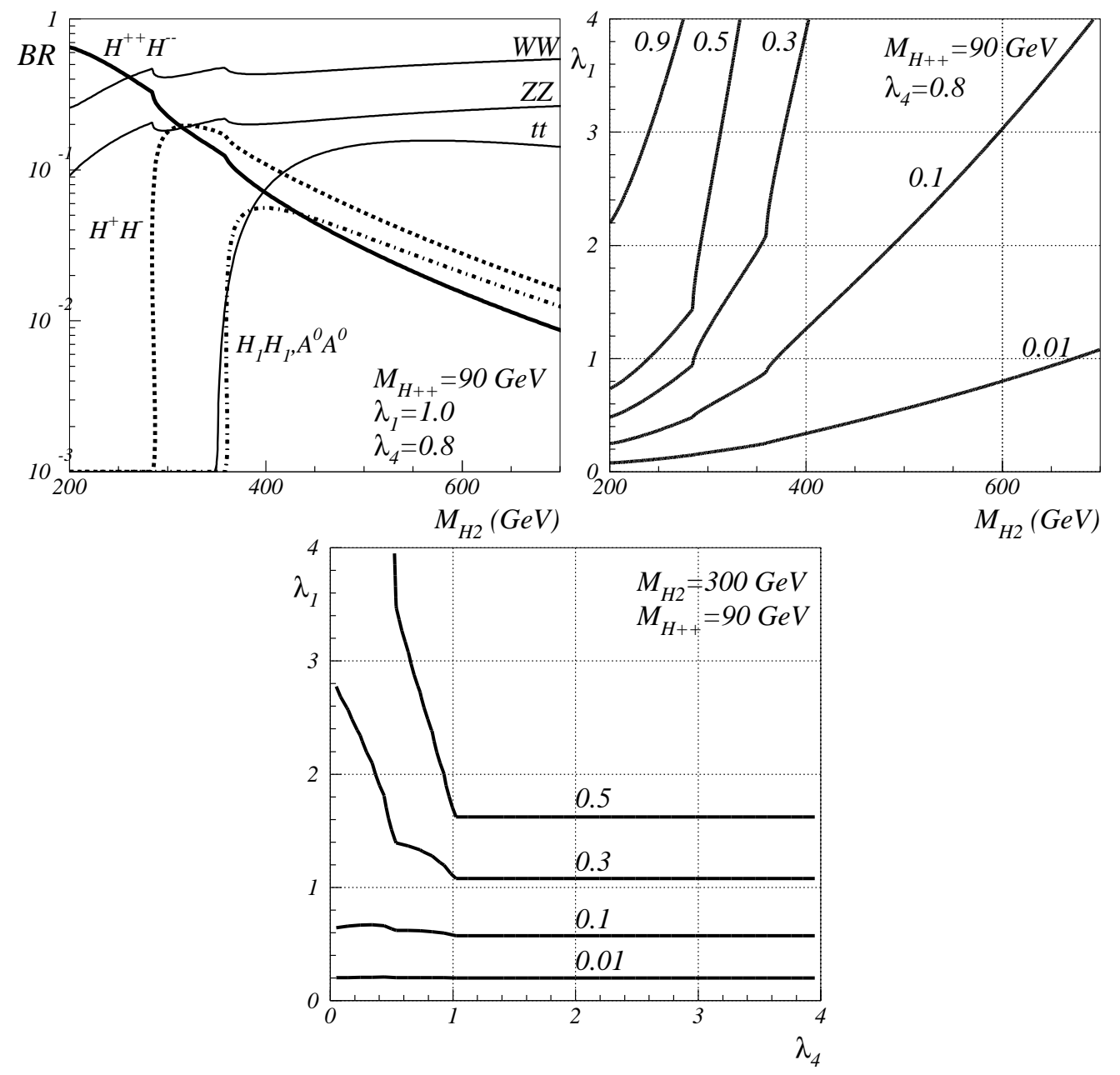

FIG. 1: Upper left panel a): branching ratios of $H_{2}$ as a function of $M_{H_{2}}$. Upper right panel b): Contours of $\mathrm{BR}\left(H_{2} \rightarrow H^{++} H^{--}\right)$in the plane $\left[M_{H_{2}}, \lambda_{1}\right]$. Lower panel c): Contours of $\mathrm{BR}\left(H_{2} \rightarrow\right.$ $H^{++} H^{--}$in the plane $\left[\lambda_{4}, \lambda_{1}\right]$. In all figures $M_{H^{ \pm \pm}}=90 \mathrm{GeV}$. In a) and b) $\lambda_{4}=0.8$, which gives $M_{H^{ \pm}}=142 \mathrm{GeV}$ and $M_{A^{0}, H_{1}}=179 \mathrm{GeV}$. In c) $M_{H_{2}}=300 \mathrm{GeV}$.

The case of $M_{H_{2}}>2 M_{H^{ \pm \pm}}$necessarily requires $M_{H_{2}} \gtrsim 200 \mathrm{GeV}$, and this mass region is now being probed for the first time at the LHC for the decay channels of $H_{2}$ to SM particles, $H_{2} \rightarrow W W$ and $H_{2} \rightarrow Z Z$. The possibility of a SM-like Higgs boson in the HTM with $M_{H_{2}}>2 M_{H^{ \pm \pm}}$and its potential impact on the direct searches for $H^{ \pm \pm}$should be clarified within the $\sqrt{s}=7 \mathrm{TeV}$ run at the LHC.

\section{Acknowledgements}

We thank Hiroaki Sugiyama and Abdesslam Arhrib for useful discussions. A.G.A was supported by a Marie Curie Incoming International Fellowship, FP7-PEOPLE-2009-IIF, Contract No. 252263. This work is supported in part by the NExT Institute. 

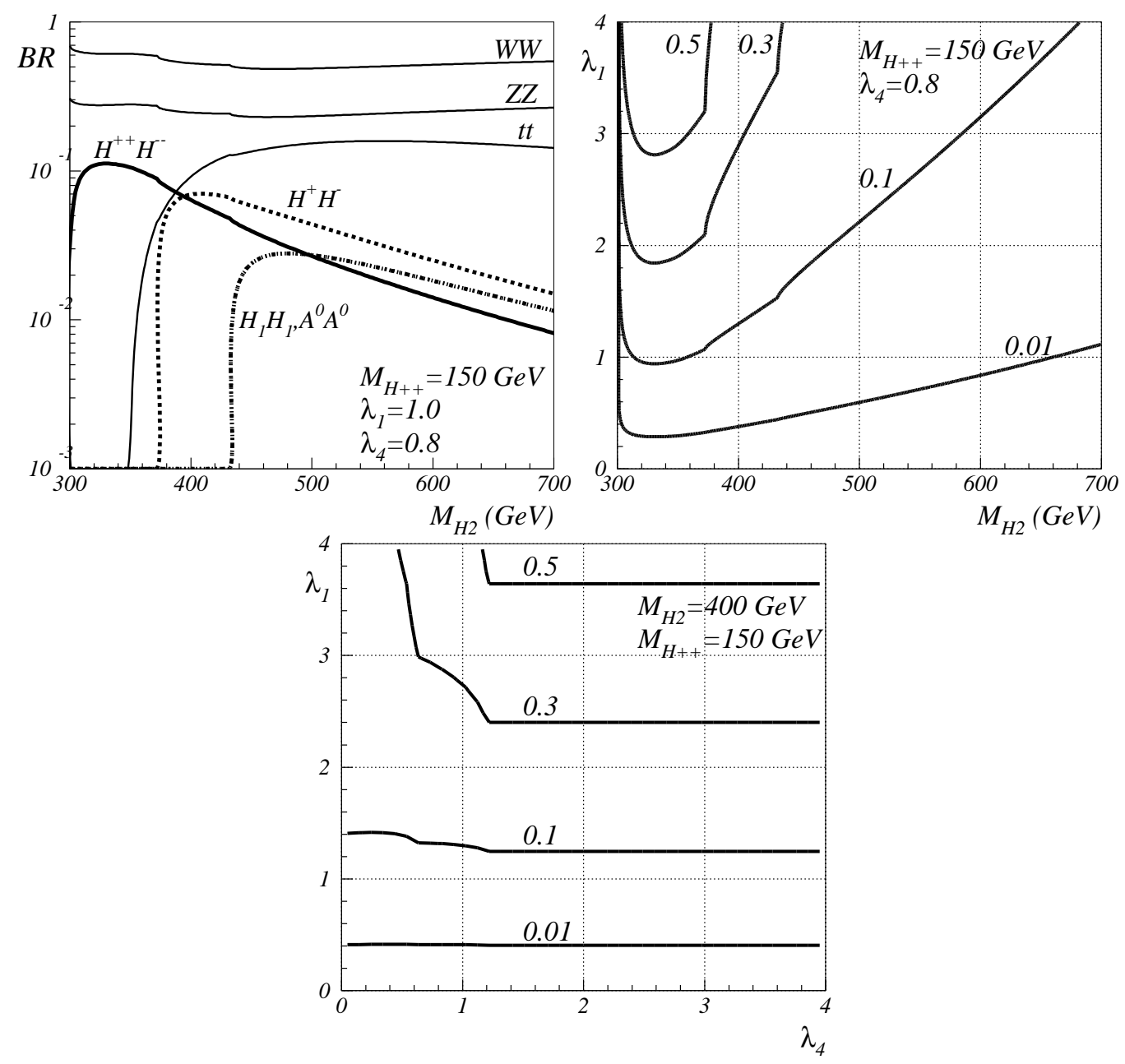

FIG. 2: Upper left panel a): branching ratios of $H_{2}$ as a function of $M_{H_{2}}$. Upper right panel b): Contours of $\mathrm{BR}\left(H_{2} \rightarrow H^{++} H^{--}\right)$in the plane $\left[M_{H_{2}}, \lambda_{1}\right]$. Lower panel c): Contours of $\mathrm{BR}\left(H_{2} \rightarrow\right.$ $H^{++} H^{--}$in the plane $\left[\lambda_{4}, \lambda_{1}\right]$. In all figures $M_{H^{ \pm \pm}}=150 \mathrm{GeV}$. In a) and b) $\lambda_{4}=0.8$, which gives $M_{H^{ \pm}}=186 \mathrm{GeV}$ and $M_{A^{0}, H_{1}}=216 \mathrm{GeV}$. In c) $M_{H_{2}}=400 \mathrm{GeV}$.

\section{Note Added}

After submission of this paper, the LHC searches for the SM Higgs boson were updated with $\mathcal{L}=1.1 \mathrm{fb}^{-1}$ [50]. For the region $M_{H_{2}}>200 \mathrm{GeV}$, both of the CMS and ATLAS collaborations use the decay channel $H_{2} \rightarrow Z Z$, with subsequent decays $Z Z \rightarrow \ell^{+} \ell^{-} \nu \nu, Z Z \rightarrow \ell^{+} \ell^{-} q \bar{q}$ and $Z Z \rightarrow \ell^{+} \ell^{-} \ell^{+} \ell^{-}$. CMS also search for $H_{2} \rightarrow W W$ with the decay mode $W W \rightarrow \ell \nu \ell \nu$, while ATLAS search for $H_{2} \rightarrow W W$ with the decay mode $W W \rightarrow \ell \nu q^{\prime} \bar{q}$. After combining the results from these four distinct channels, both collaborations exclude at $95 \%$ c.l the mass range $295 \mathrm{GeV}<M_{H_{2}}<450 \mathrm{GeV}$. This does not preclude a sizeable value of $R$ in the HTM, e.g. from fig. 6re, one can see that $7>R>1$ in the interval $450 \mathrm{GeV}<M_{H_{2}}<600 \mathrm{GeV}$, for $\lambda_{1}=4$ and $\sqrt{s}=7 \mathrm{TeV}$ (and not including the 

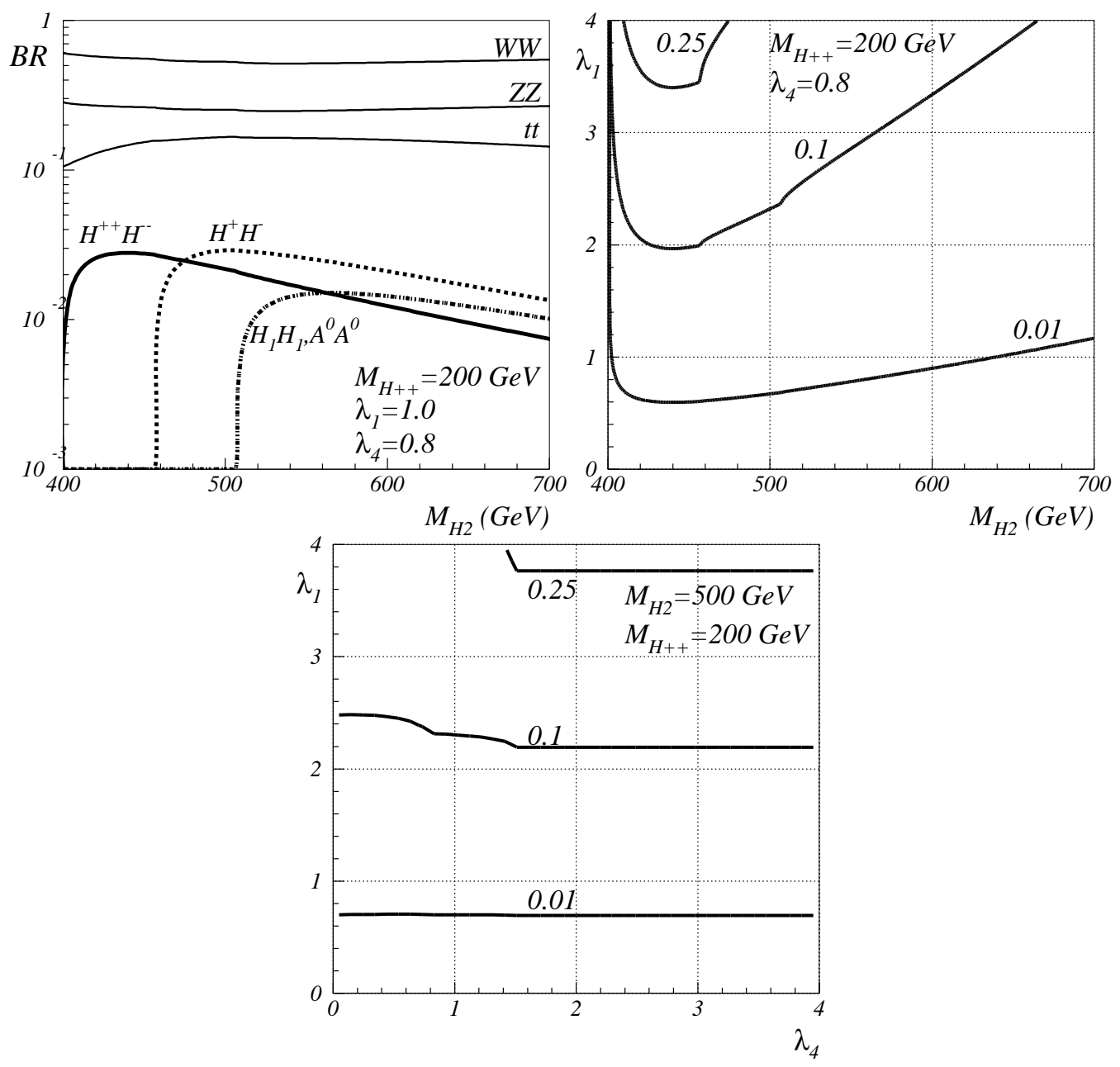

FIG. 3: Upper left panel a): branching ratios of $H_{2}$ as a function of $M_{H_{2}}$. Upper right panel b): Contours of $\mathrm{BR}\left(H_{2} \rightarrow H^{++} H^{--}\right)$in the plane $\left[M_{H_{2}}, \lambda_{1}\right]$. Lower panel c): Contours of $\mathrm{BR}\left(H_{2} \rightarrow\right.$ $H^{++} H^{--}$in the plane $\left[\lambda_{4}, \lambda_{1}\right]$. In all figures $M_{H^{ \pm \pm}}=200 \mathrm{GeV}$. In a) and b) $\lambda_{4}=0.8$, which gives $M_{H^{ \pm}}=228 \mathrm{GeV}$ and $M_{A^{0}, H_{1}}=253 \mathrm{GeV}$. In c) $M_{H_{2}}=500 \mathrm{GeV}$.

enhancement from the QCD $K$ factor).

[1] Y. Fukuda et al. [Super-Kamiokande Collaboration], Phys. Rev. Lett. 81, 1562 (1998).

[2] Y. Kuno and Y. Okada, Rev. Mod. Phys. 73, 151 (2001); M. Raidal et al., Eur. Phys. J. C 57, 13 (2008).

[3] W. Konetschny and W. Kummer, Phys. Lett. B 70, 433 (1977).

[4] R. N. Mohapatra and G. Senjanovic, Phys. Rev. Lett. 44, 912 (1980).

[5] M. Magg and C. Wetterich, Phys. Lett. B 94, 61 (1980); G. Lazarides, Q. Shafi and C. Wetterich, Nucl. Phys. B 181, 287 (1981).

[6] J. Schechter and J. W. F. Valle, Phys. Rev. D 22, 2227 (1980).

[7] T. P. Cheng and L. F. Li, Phys. Rev. D 22, 2860 (1980). 

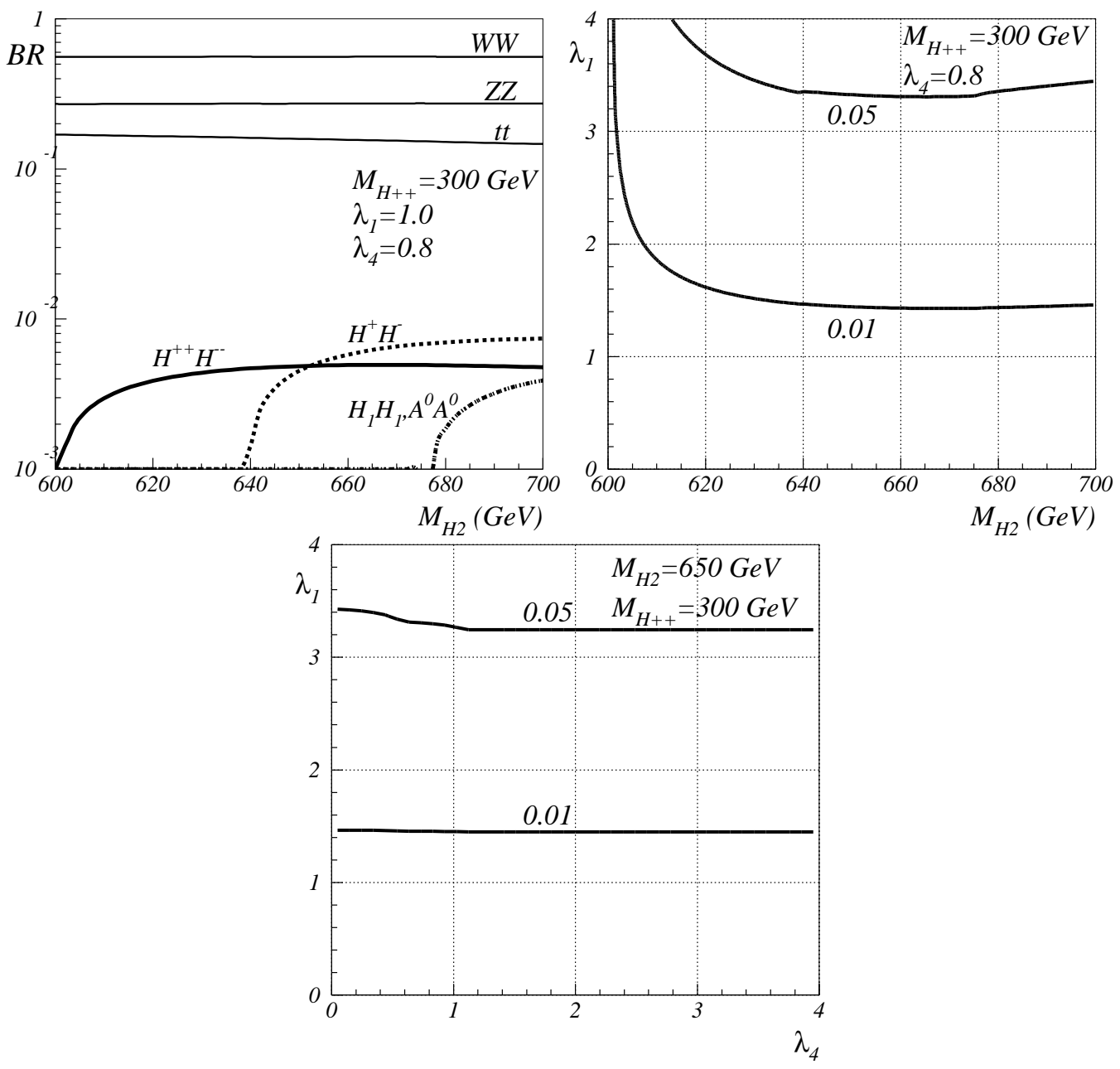

FIG. 4: Upper left panel a): branching ratios of $H_{2}$ as a function of $M_{H_{2}}$. Upper right panel b): Contours of $\mathrm{BR}\left(H_{2} \rightarrow H^{++} H^{--}\right)$in the plane $\left[M_{H_{2}}, \lambda_{1}\right]$. Lower panel c): Contours of $\mathrm{BR}\left(H_{2} \rightarrow\right.$ $H^{++} H^{--}$in the plane $\left[\lambda_{4}, \lambda_{1}\right]$. In all figures $M_{H^{ \pm \pm}}=300 \mathrm{GeV}$. In a) and b) $\lambda_{4}=0.8$, which gives $M_{H^{ \pm}}=320 \mathrm{GeV}$ and $M_{A^{0}, H_{1}}=338 \mathrm{GeV}$. In c) $M_{H_{2}}=650 \mathrm{GeV}$.

[8] V. D. Barger, H. Baer, W. Y. Keung and R. J. N. Phillips, Phys. Rev. D 26, 218 (1982).

[9] J. F. Gunion, J. Grifols, A. Mendez, B. Kayser and F. I. Olness, Phys. Rev. D 40, 1546 (1989); J. F. Gunion, C. Loomis and K. T. Pitts, eConf C960625, LTH096 (1996) arXiv:hep-ph/9610237.

[10] M. Muhlleitner and M. Spira, Phys. Rev. D 68, 117701 (2003).

[11] T. Han, B. Mukhopadhyaya, Z. Si and K. Wang, Phys. Rev. D 76, 075013 (2007).

[12] K. Huitu, J. Maalampi, A. Pietila and M. Raidal, Nucl. Phys. B 487, 27 (1997).

[13] B. Dion, T. Gregoire, D. London, L. Marleau and H. Nadeau, Phys. Rev. D 59, 075006 (1999).

[14] A. G. Akeroyd and M. Aoki, Phys. Rev. D 72, 035011 (2005).

[15] D. E. Acosta et al. [CDF Collaboration], Phys. Rev. Lett. 93, 221802 (2004).

[16] V. M. Abazov et al. [D0 Collaboration], Phys. Rev. Lett. 93, 141801 (2004).

[17] V. M. Abazov et al. [D0 Collaboration], Phys. Rev. Lett. 101, 071803 (2008).

[18] T. Aaltonen et al. [The CDF Collaboration], Phys. Rev. Lett. 101, 121801 (2008). 

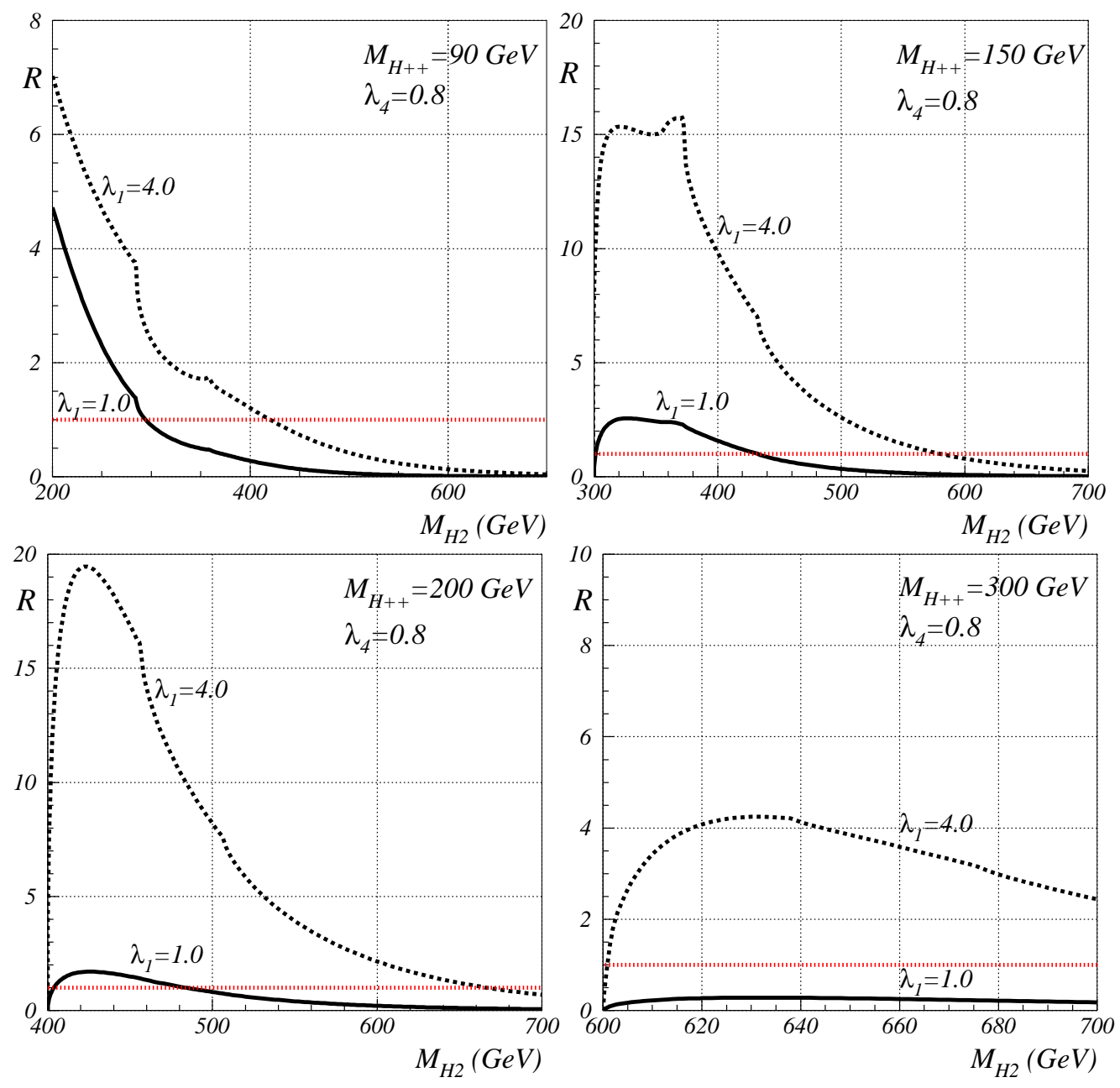

FIG. 5: The ratio $R=\sigma\left(g g \rightarrow H_{2}\right) \times \mathrm{BR}\left(H_{2} \rightarrow H^{++} H^{--}\right) / \sigma\left(q \bar{q} \rightarrow H^{++} H^{--}\right)$at the LHC (with $\sqrt{s}=14 \mathrm{TeV})$ as a function of $M_{H_{2}}$. The two curves are for $\lambda_{1}=1$ and $\lambda_{1}=4$, with $\lambda_{4}=0.8$. We take $M_{H^{ \pm \pm}}=90 \mathrm{GeV}$ in panel (a), $M_{H^{ \pm \pm}}=150 \mathrm{GeV}$ in panel (b), $M_{H^{ \pm \pm}}=200 \mathrm{GeV}$ in panel (c), and $M_{H^{ \pm \pm}}=300 \mathrm{GeV}$ in panel (d). The horizontal line shows $R=1$.

[19] E. Ma, M. Raidal and U. Sarkar, Phys. Rev. Lett. 85, 3769 (2000); E. Ma, M. Raidal and U. Sarkar, Nucl. Phys. B 615, 313 (2001).

[20] E. J. Chun, K. Y. Lee and S. C. Park, Phys. Lett. B 566, 142 (2003).

[21] J. Garayoa and T. Schwetz, JHEP 0803, 009 (2008).

[22] A. G. Akeroyd, M. Aoki and H. Sugiyama, Phys. Rev. D 77, 075010 (2008).

[23] M. Kadastik, M. Raidal and L. Rebane, Phys. Rev. D 77, 115023 (2008).

[24] P. Fileviez Perez, T. Han, G. y. Huang, T. Li and K. Wang, Phys. Rev. D 78, 015018 (2008).

[25] F. del Aguila and J. A. Aguilar-Saavedra, Nucl. Phys. B 813, 22 (2009).

[26] A. G. Akeroyd and C. W. Chiang, Phys. Rev. D 80, 113010 (2009).

[27] G. Azuelos, K. Benslama and J. Ferland, J. Phys. G 32, 73 (2006).

[28] T. Rommerskirchen and T. Hebbeker, J. Phys. G 34, N47 (2007).

[29] A. G. Akeroyd, C. W. Chiang and N. Gaur, JHEP 1011, 005 (2010).

[30] CMS Collaboration, CMS PAS HIG-11-001 (March 2011). 

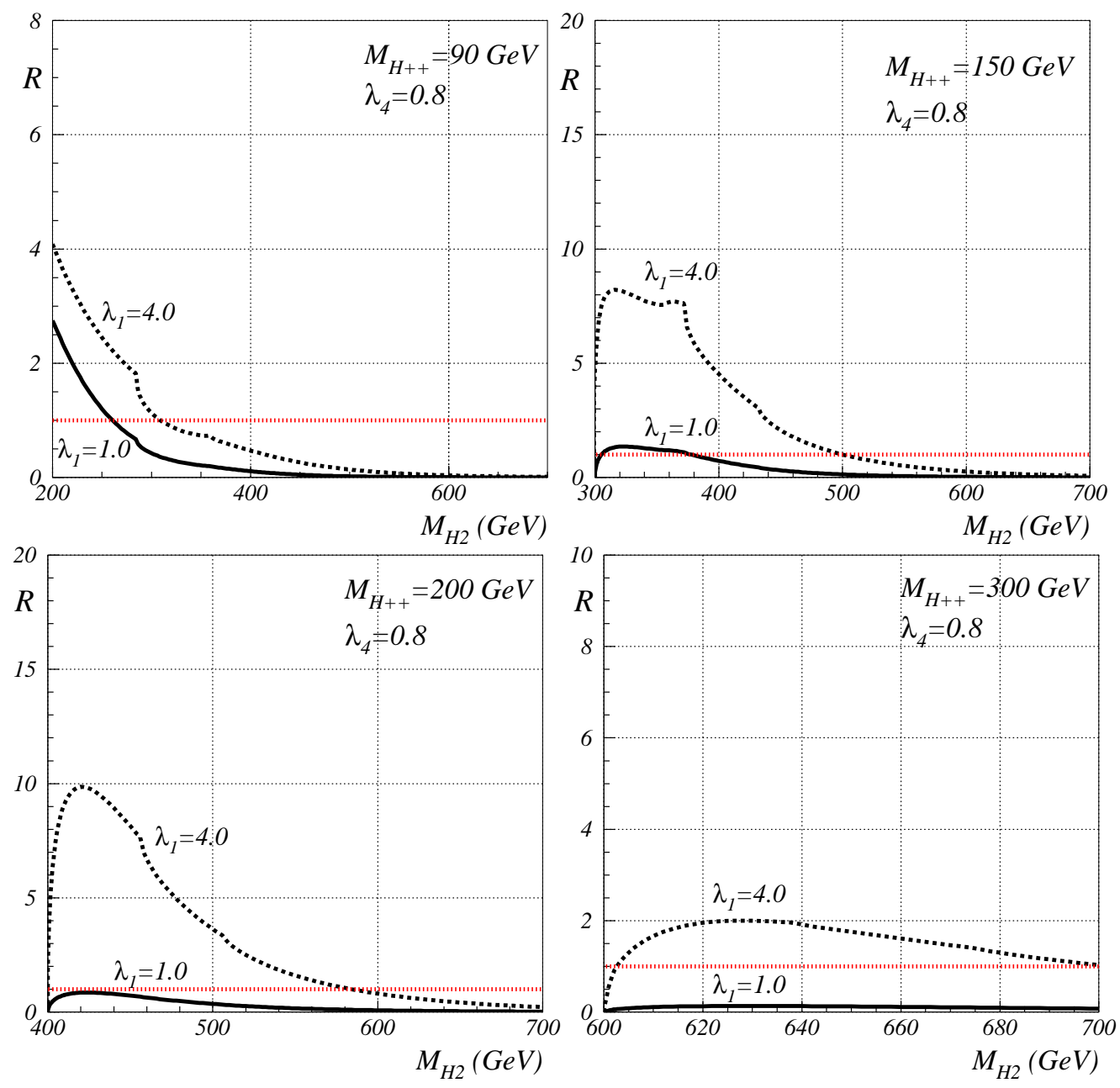

FIG. 6: The ratio $R=\sigma\left(g g \rightarrow H_{2}\right) \times \mathrm{BR}\left(H_{2} \rightarrow H^{++} H^{--}\right) / \sigma\left(q \bar{q} \rightarrow H^{++} H^{--}\right)$at the LHC (with $\sqrt{s}=7 \mathrm{TeV})$ as a function of $M_{H_{2}}$. The two curves are for $\lambda_{1}=1$ and $\lambda_{1}=4$, with $\lambda_{4}=0.8$. We take $M_{H^{ \pm \pm}}=90 \mathrm{GeV}$ in panel (a), $M_{H^{ \pm \pm}}=150 \mathrm{GeV}$ in panel (b), $M_{H^{ \pm \pm}}=200 \mathrm{GeV}$ in panel (c), and $M_{H^{ \pm \pm}}=300 \mathrm{GeV}$ in panel (d). The horizontal line shows $R=1$.

[31] P. Dey, A. Kundu and B. Mukhopadhyaya, J. Phys. G 36, 025002 (2009).

[32] A. G. Akeroyd and C. W. Chiang, Phys. Rev. D 81, 115007 (2010).

[33] A. Arhrib, R. Benbrik, M. Chabab, G. Moultaka, M. C. Peyranere, L. Rahili and J. Ramadan, arXiv:1105.1925 [hep-ph].

[34] ATLAS Collaboration, arXiv:1106.2748 [hep-ex].

[35] S. Chatrchyan et al. [CMS Collaboration], Phys. Lett. B 699, 25 (2011).

[36] G. B. Gelmini and M. Roncadelli, Phys. Lett. B 99, 411 (1981).

[37] J. Schechter and J. W. F. Valle, Phys. Rev. D 25, 774 (1982); M. A. Diaz, M. A. GarciaJareno, D. A. Restrepo and J. W. F. Valle, Nucl. Phys. B 527, 44 (1998); A. G. Akeroyd, M. A. Diaz, M. A. Rivera, D. Romero, Phys. Rev. D83, 095003 (2011).

[38] S. K. Majee and N. Sahu, Phys. Rev. D 82, 053007 (2010).

[39] J. F. Gunion, R. Vega and J. Wudka, Phys. Rev. D 42, 1673 (1990); R. Vega and D. A. Dicus, Nucl. Phys. B 329, 533 (1990); J. Maalampi and N. Romanenko, Phys. Lett. B 532, 202 

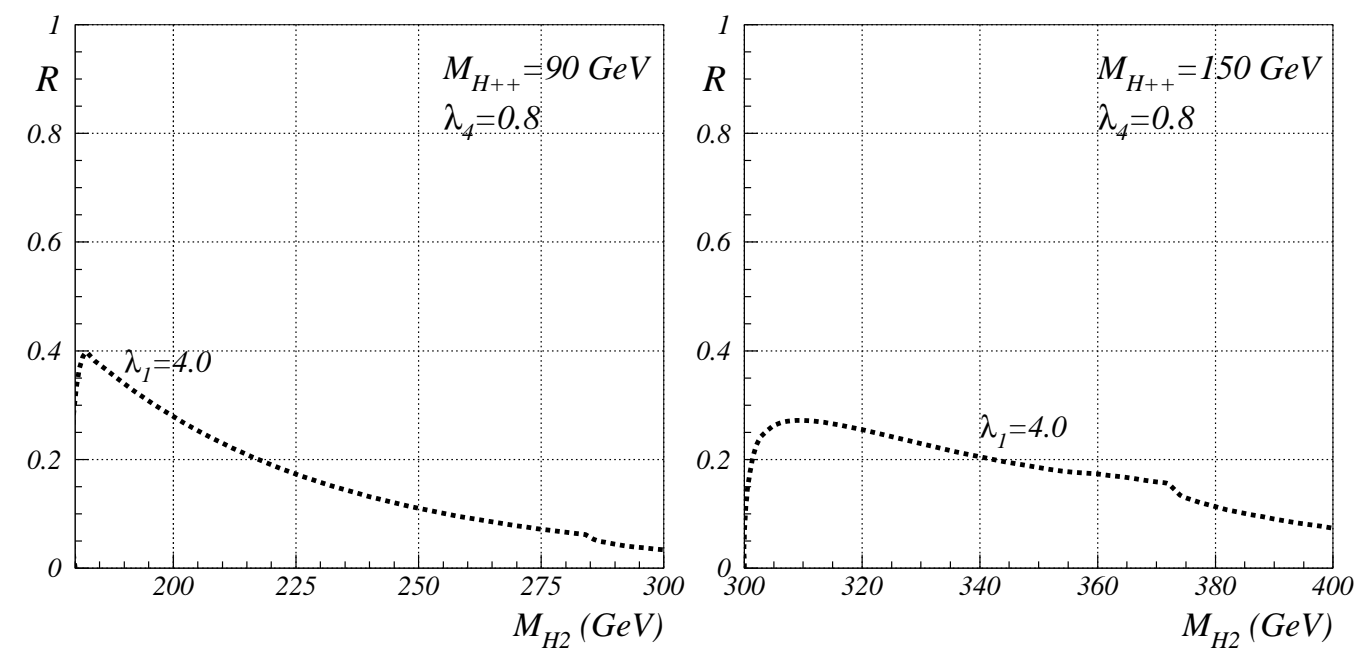

FIG. 7: The ratio $R=\sigma\left(g g \rightarrow H_{2}\right) \times \mathrm{BR}\left(H_{2} \rightarrow H^{++} H^{--}\right) / \sigma\left(q \bar{q} \rightarrow H^{++} H^{--}\right)$at the Tevatron (with $\sqrt{s}=1.96 \mathrm{TeV}$ ) as a function of $M_{H_{2}}$. The curves are for $\lambda_{1}=4$ and $\lambda_{4}=0.8$. We take $M_{H^{ \pm \pm}}=90 \mathrm{GeV}$ in panel (a), $M_{H^{ \pm \pm}}=150 \mathrm{GeV}$ in panel (b).

(2002).

[40] T. Blank and W. Hollik, Nucl. Phys. B 514, 113 (1998); J. R. Forshaw, D. A. Ross, B. E. White, JHEP 0110, 007 (2001); J. R. Forshaw, A. Sabio Vera, B. E. White, JHEP 0306, 059 (2003); P. H. Chankowski, S. Pokorski and J. Wagner, Eur. Phys. J. C 50, 919 (2007); M. C. Chen, S. Dawson and C. B. Jackson, Phys. Rev. D 78, 093001 (2008).

[41] M. C. Chen, S. Dawson and T. Krupovnickas, Phys. Rev. D 74, 035001 (2006).

[42] S. de Visscher, J. M. Gerard, M. Herquet, V. Lemaitre and F. Maltoni, JHEP 0908, 042 (2009).

[43] J. Abdallah et al. [DELPHI Collaboration], Phys. Lett. B 552, 127 (2003) arXiv:hep-ex/0303026]; G. Abbiendi et al. [OPAL Collaboration], Phys. Lett. B 526, 221 (2002); P. Achard et al. [L3 Collaboration], Phys. Lett. B 576, 18 (2003).

[44] T. Aaltonen et al. [CDF Collaboration], arXiv:1108.0101 [hep-ex].

[45] J. Pumplin, D. R. Stump, J. Huston, H. L. Lai, P. M. Nadolsky and W. K. Tung, JHEP 0207, 012 (2002); D. Stump, J. Huston, J. Pumplin, W. K. Tung, H. L. Lai, S. Kuhlmann and J. F. Owens, JHEP 0310, 046 (2003).

[46] H. M. Georgi, S. L. Glashow, M. E. Machacek and D. V. Nanopoulos, Phys. Rev. Lett. 40, 692 (1978); M. Spira, A. Djouadi, D. Graudenz and P. M. Zerwas, Nucl. Phys. B 453, 17 (1995).

[47] A. Djouadi, Phys. Rept. 457, 1 (2008).

[48] A. G. Akeroyd, H. Sugiyama, arXiv:1105.2209 [hep-ph]].

[49] V. Rentala, W. Shepherd, S. Su, arXiv:1105.1379 [hep-ph]].

[50] Talk by W. Murray at the International Europhysics Conference on High Energy Physics, Grenoble, France, July 21-27 2011. 

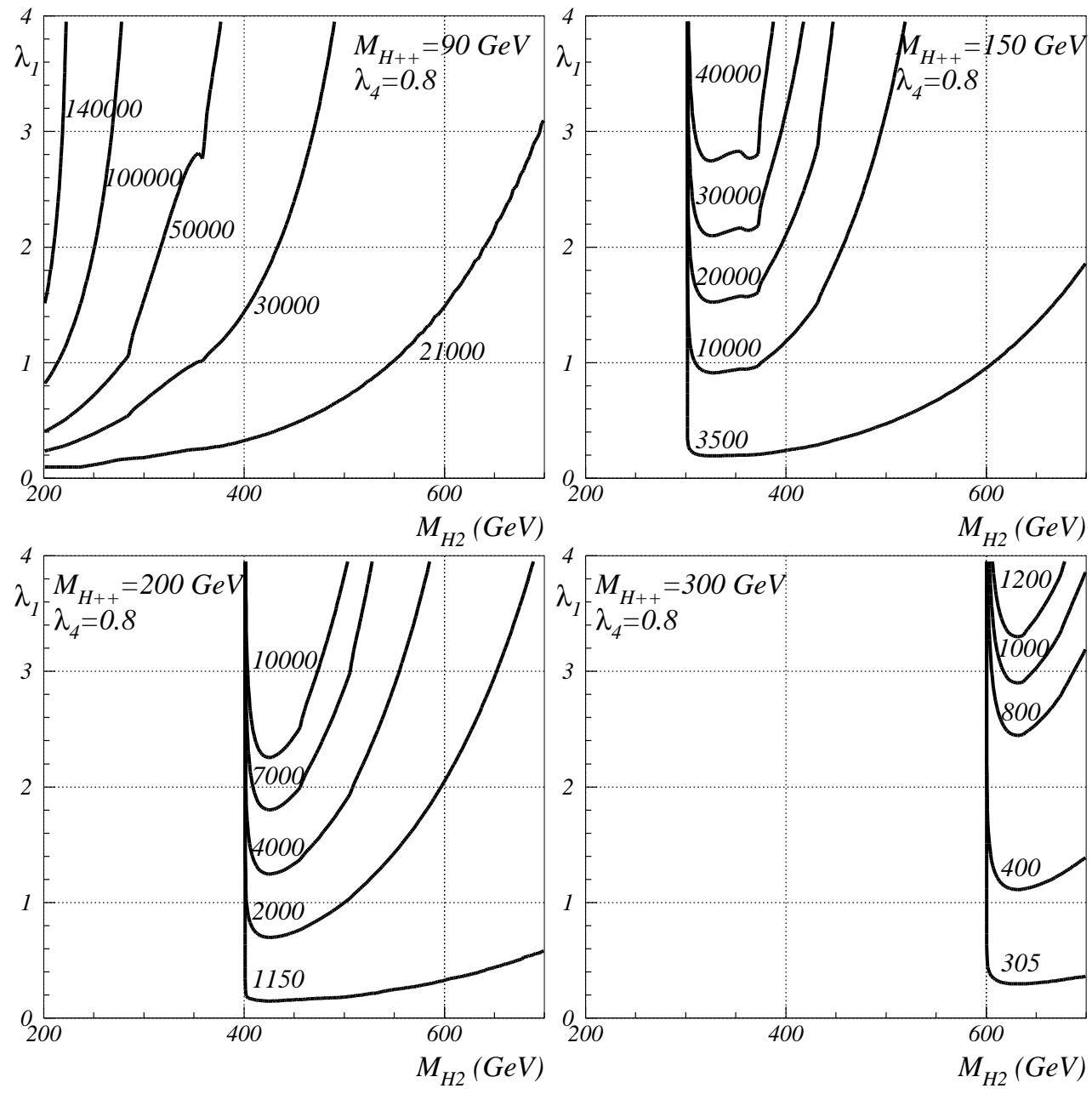

FIG. 8: The number of $H^{++} H^{--}$events (assuming $\operatorname{BR}\left(H^{ \pm \pm} \rightarrow \mu^{ \pm} \mu^{ \pm}\right)=100 \%$ ) at the LHC with $\sqrt{s}=14 \mathrm{TeV}$ and $\mathcal{L}=30 \mathrm{fb}^{-1}$ in the plane $\left[M_{H_{2}}, \lambda_{1}\right]$. We take $M_{H^{ \pm \pm}}=90 \mathrm{GeV}$ in panel (a), $M_{H^{ \pm \pm}}=150 \mathrm{GeV}$ in panel (b), $M_{H^{ \pm \pm}}=200 \mathrm{GeV}$ in panel (c) and $M_{H^{ \pm \pm}}=300 \mathrm{GeV}$ in panel (d). In all figures $\lambda_{4}=0.8$. The number of events for $q \bar{q} \rightarrow \gamma, Z \rightarrow H^{++} H^{--}$alone is 20500, 3270, 1130 and 299 in (a),(b),(c) and (d), respectively. 

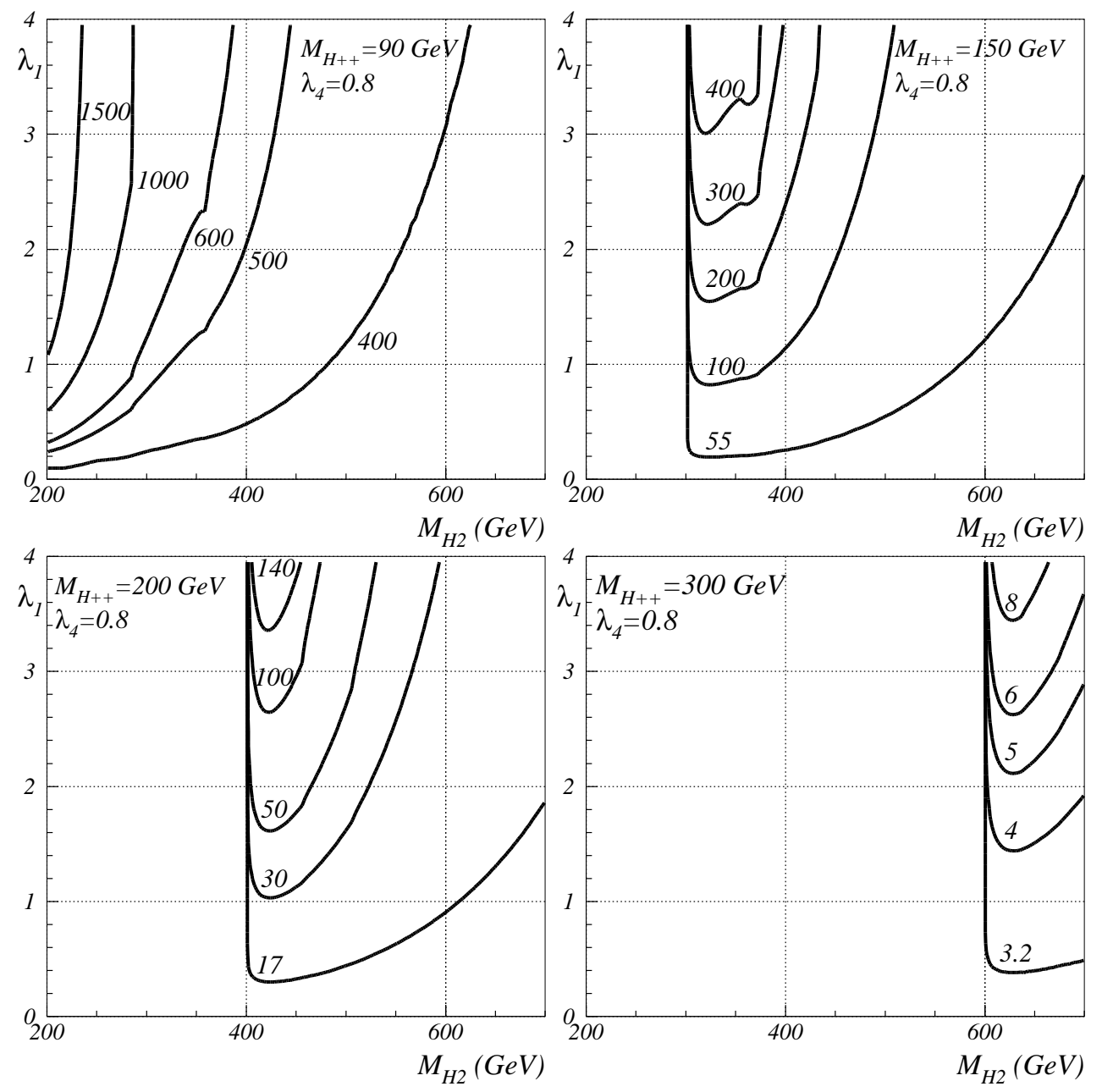

FIG. 9: The number of $H^{++} H^{--}$events (assuming $\mathrm{BR}\left(H^{ \pm \pm} \rightarrow \mu^{ \pm} \mu^{ \pm}\right)=100 \%$ ) at the LHC with $\sqrt{s}=7 \mathrm{TeV}$ and $\mathcal{L}=2 \mathrm{fb}^{-1}$ in the plane $\left[M_{H_{2}}, \lambda_{1}\right]$. We take $M_{H^{ \pm \pm}}=90 \mathrm{GeV}$ in panel (a), $M_{H^{ \pm \pm}}=150 \mathrm{GeV}$ in panel (b), $M_{H^{ \pm \pm}}=200 \mathrm{GeV}$ in panel (c) and $M_{H^{ \pm \pm}}=300 \mathrm{GeV}$ in panel (d). In all figures $\lambda_{4}=0.8$. The number of events for $q \bar{q} \rightarrow \gamma, Z \rightarrow H^{++} H^{--}$alone is 390, 53, 16, 3 in (a),(b),(c) and (d), respectively. 